\title{
Wind-driven mixing below the oceanic mixed layer
}

Article

Published Version

Grant, A. L. M. and Belcher, S. (2011) Wind-driven mixing below the oceanic mixed layer. Journal of Physical

Oceanography, 41 (8). pp. 1556-1575. ISSN 0022-3670 doi: https://doi.org/10.1175/JPO-D-10-05020.1 Available at https://centaur.reading.ac.uk/26959/

It is advisable to refer to the publisher's version if you intend to cite from the work. See Guidance on citing.

Published version at: http://dx.doi.org/10.1175/JPO-D-10-05020.1

To link to this article DOI: http://dx.doi.org/10.1175/JPO-D-10-05020.1

Publisher: American Meteorological Society

All outputs in CentAUR are protected by Intellectual Property Rights law, including copyright law. Copyright and IPR is retained by the creators or other copyright holders. Terms and conditions for use of this material are defined in the End User Agreement.

\section{www.reading.ac.uk/centaur}

\section{CentAUR}

Central Archive at the University of Reading

Reading's research outputs online 


\title{
Wind-Driven Mixing below the Oceanic Mixed Layer
}

\author{
Alan L. M. Grant And StePhen E. Belcher \\ University of Reading, Reading, United Kingdom
}

(Manuscript received 14 December 2010, in final form 23 March 2011)

\begin{abstract}
This study describes the turbulent processes in the upper ocean boundary layer forced by a constant surface stress in the absence of the Coriolis force using large-eddy simulation. The boundary layer that develops has a two-layer structure, a well-mixed layer above a stratified shear layer. The depth of the mixed layer is approximately constant, whereas the depth of the shear layer increases with time. The turbulent momentum flux varies approximately linearly from the surface to the base of the shear layer.

There is a maximum in the production of turbulence through shear at the base of the mixed layer. The magnitude of the shear production increases with time. The increase is mainly a result of the increase in the turbulent momentum flux at the base of the mixed layer due to the increase in the depth of the boundary layer. The length scale for the shear turbulence is the boundary layer depth. A simple scaling is proposed for the magnitude of the shear production that depends on the surface forcing and the average mixed layer current. The scaling can be interpreted in terms of the divergence of a mean kinetic energy flux.

A simple bulk model of the boundary layer is developed to obtain equations describing the variation of the mixed layer and boundary layer depths with time. The model shows that the rate at which the boundary layer deepens does not depend on the stratification of the thermocline. The bulk model shows that the variation in the mixed layer depth is small as long as the surface buoyancy flux is small.
\end{abstract}

\section{Introduction}

The action of the wind on the sea generates surface waves and turbulence within the upper ocean. Turbulent mixing leads to the development of a mixed layer (ML) with small vertical gradients in temperature and salinity above the stratified ocean beneath. This simple structure is the basis for mixed layer models of the ocean surface boundary layer (OSBL; Niller and Kraus 1977; Garwood 1977). In these models, the base of the mixed layer is assumed to be marked by a change in buoyancy over a transition layer whose depth is small compared to the depth of the mixed layer. Through the action of turbulence, water in the thermocline is entrained into the mixed layer, modifying its properties and causing it to deepen.

Contrary to this idealized picture, the transition layer between the mixed layer and underlying thermocline is often observed to be relatively thick (Johnston and Rudnick 2009). Observations show that the transition

Corresponding author address: Alan L. M. Grant, Department of Meteorology, University of Reading, P.O. Box 243, Reading RG6 6BB, United Kingdom.

E-mail: a.1.m.grant@reading.ac.uk layer is turbulent and that the turbulence may be coupled to the surface forcing. Examples of the coupling between surface forcing and turbulence in the transition region are as follows:

- A significant fraction of the wind-driven Ekman transports are observed to occur below the base of the mixed layer (Lentz 1992; Rudnick 2003), implying that a significant fraction of the surface stress penetrates below the mixed layer.

- During the passage of storms, strong turbulent mixing below the base of the mixed layer is observed when the turning of the surface winds due to storm motion occurs close to the inertial frequency (Large and Crawford 1995). Significant exchange of heat between the mixed layer and thermocline occur during such events, as shown, for example, by the cold wakes left after the passage of hurricanes (Zedler et al. 2002).

- In the eastern equatorial Pacific, turbulence levels below the base of the nocturnal mixed layer exhibit a marked diurnal cycle (Lien et al. 2002; Moum et al. 2009). The intensity of the turbulence is greatest at night, when the mixed layer is deepest. This phenomenon is referred to as deep cycle turbulence. 
The deep cycle turbulence in the eastern Pacific occurs within the shear layer above the equatorial undercurrent and is the best observed example of coupling between turbulence below the mixed layer and surface forcing (Lien et al. 1995). The mechanism responsible for the coupling between the turbulence and surface forcing is still not fully understood. Observations show evidence of both waves and shear instabilities in the region below the mixed layer, but it is not clear whether breaking of the waves is responsible for the turbulence (Gregg et al. 1985) or whether the turbulence and waves are a consequence of shear instability (Moum et al. 1992; Sun et al. 1998).

Skyllingstad et al. (2000) used large-eddy simulation (LES) to study resonant wind-driven mixing. Turbulent kinetic energy (TKE) budgets showed a maximum in the production of turbulence due to the current shear at the base of the mixed layer rather than at the surface. The shear production of turbulence extended through the mixed layer as well as the stratified shear layer (SSL) below.

The need to parameterize the effects of turbulence below the mixed layer has long been recognized (Price et al. 1986; Large et al. 1994; Kantha and Clayson 1994), but it is still not clear how this should be done. In the $K$-profile parameterization (KPP) scheme described by Large et al. (1994), the depth of the boundary layer is diagnosed using a bulk Richardson number, which allows the boundary layer to be deeper than the mixed layer. When this occurs, the turbulence in and below the mixed layer is assumed to scale in the same way. The KPP scheme has been shown to model the effects of both resonant wind-driven mixing (Large and Crawford 1995) and the diurnal signal of deep cycle mixing (Large and Gent 1999).

Kantha and Clayson (1994) treated the mixed layer and transition layer separately using a local Richardson number scheme to represent mixing by turbulence in the transition zone. In this view the turbulence in the stratified region may arise from a number of sources, such as internal waves or intermittent Kelvin-Helmholtz instability within the stratified layer. A problem with this approach is that the Richardson number scheme uses a dimensional value for the diffusivity at small Richardson numbers, a feature that has been criticized by Mellor (2001) and Zaron and Moum (2009). In addition, this treatment does not provide an explicit connection between the surface forcing and turbulent mixing.

It is not clear whether all of the phenomena listed above have a single explanation, given that they occur in different large-scale contexts. However, LES of resonant mixing (Skyllingstad et al. 2000) and deep cycle turbulence (Wang et al. 1998) suggest that shear production of turbulence at the base of the mixed layer may be a common feature. This study uses LES to investigate the evolution of the upper ocean boundary layer forced by a constant stress at the surface. The problem is simplified by taking the Coriolis parameter to be zero to eliminate the problem of inertial oscillations. These simulations correspond most closely to the problem of resonant wind-driven mixing (Large and Crawford 1995; Skyllingstad et al. 2000) in which currents and surface stress remain aligned. The study addresses the following questions:

(i) How is the intensity of the turbulence below the mixed layer related to the surface forcing?

(ii) What is the nature of the interaction between the mixed layer and transition layer?

\section{Details of large-eddy simulations}

The simulations were carried out using the Met Office large-eddy model (LEM), modified to simulate the upper ocean boundary layer. In this model, a leapfrog time step is used together with the second-order accurate advection scheme described by Piacsek and Williams (1970). The subgrid scheme uses a stability-dependent Smagorinsky diffusivity (Brown et al. 1994). The atmospheric version of the large-eddy model is described in detail by Shutts and Gray (1994), and the modifications to simulate the oceanic mixed layer are described in Grant and Belcher (2009).

For the present simulations, the horizontal resolution was $2 \mathrm{~m}$ with a domain size of $256 \times 256$ points. In the vertical, there were 152 points with a spacing of $0.8 \mathrm{~m}$. Below $80 \mathrm{~m}$, a sponge layer was imposed to damp out waves.

Results from three simulations are described. All of the simulations are started from rest and forced using a constant surface stress of $0.037 \mathrm{~N} \mathrm{~m}^{-2}$. The surface buoyancy flux is zero in all simulations. The initial conditions for simulation A are based on the simulations described by McWilliams et al. (1997). The initial mixed layer depth is $33 \mathrm{~m}$, with constant temperature gradient of $0.001 \mathrm{~K} \mathrm{~m}^{-1}$ below this. Simulations B and C are the same as simulation $\mathrm{A}$, but with an initial mixed layer depth of $25 \mathrm{~m}$ in simulation $\mathrm{B}$, whereas in simulation $\mathrm{C}$ the temperature gradient below the mixed is set to $0.0005 \mathrm{~K} \mathrm{~m}^{-1}$.

In all simulations, the Coriolis parameter is zero. This means that the surface stress and currents remain aligned and also that there is no steady state. Because the mean flow does not reach a steady state, the forcing applied in the simulation can be weak so that the mean flow, in particular the boundary layer depth, evolves slowly. The slow evolution means that it is possible to 

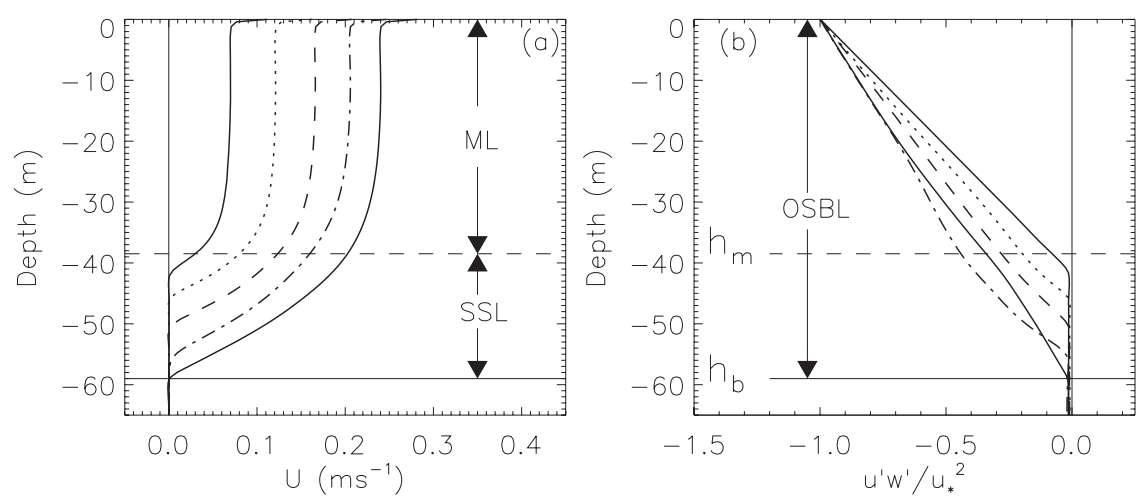

FIG. 1. Evolution with time of (a) current velocity and (b) stress profiles for simulation A. Profiles are plotted every $30000 \mathrm{~s}$. The solid curves are the first profile and the dashed-dotteddotted-dotted curve is the last. The layers referred to in the text are marked: ML, SSL, and OSBL. The depths of the ML $\left(h_{m}\right)$ and the OSBL $\left(h_{b}\right)$ are also marked.

use relatively long averaging times to calculate turbulent statistics, reducing statistical sampling errors. All simulations were allowed to spin up for $30000 \mathrm{~s}$. After the spinup period, diagnostics were output every $10000 \mathrm{~s}$, with turbulence statistics averaged over $10000 \mathrm{~s}$.

Observational evidence and work with LES have suggested that Langmuir turbulence is the dominant form of wind-driven turbulence in the mixed layer (Thorpe 2004). The present simulations include the effects of Stokes drift associated with surface waves. As in the study of Skyllingstad et al. (2000), the effects of Stokes drift on mixed layer turbulence is represented through the Craik-Leibovich vortex force (Craik and Leibovich 1976). An advantage of including the effects of surface waves on mixed layer turbulence is that the production of Langmuir turbulence through the shear in the Stokes drift and the generation of turbulence by the current shear can be clearly distinguished.

\section{Results}

Figures 1a,b show the evolution of the mean current and stress profiles for simulation A. Unsurprisingly, the velocity increases with time because of the imposed surface stress. Above $35 \mathrm{~m}$, the mean current is approximately constant with depth, whereas below $35 \mathrm{~m}$ a layer marked by shear deepens with time. The turbulent stress (Fig. 1b) decreases approximately linearly with depth between the surface and the base of the shear layer. There is some variability in the shape of the stress profiles, but this appears to be due to turbulent fluctuations.

Nondimensional stress profiles from the three simulations are shown in Fig. 2a as a function of $z / h_{b}$, where $z$ is depth and $h_{b}$ is the depth of the base of the shear layer. The variation of the turbulent stress profiles with depth is not quite linear, with the maximum deviation from a linear profile occurring around the base of the mixed layer. Close examination of Fig. 2a shows that, at depths greater than $h_{b}$, the stress is nonzero, although the magnitude is small. This is due to waves generated in the shear layer, which is discussed further in section 8 . The waves are absorbed in the damping layer at the base of the model leading to a small acceleration of the flow in this region.

Figure $2 \mathrm{~b}$ shows the turbulent momentum flux $\rho_{w} \overline{u^{\prime} w_{m}^{\prime}}$ at the base of the mixed layer as a function of $u_{*}^{2}\left(1-h_{m} / h_{b}\right)$, where $h_{m}$ is the depth of the mixed layer and $\rho_{w}$ is the density of seawater. The line in Fig. 2b shows that $-\overline{u^{\prime} w_{m}^{\prime}} \approx 1.15 u_{*}^{2}\left(1-h_{m} / h_{b}\right)$. If the boundary layer depth were constant, the stress profile would be linear, assuming that the shape of the current profile remained the same. The nonlinear stress profile in the present simulations is a consequence of the deepening of the boundary layer. Water that is incorporated into the boundary layer as it deepens must be accelerated, acting as a drag on the stratified shear layer. This drag is balanced by a transfer of additional momentum from the mixed layer to the shear layer.

The variation in temperature profiles with time is shown in Fig. 3a. Above $35 \mathrm{~m}$, the temperature is approximately constant with depth and cools slightly with time. Below $35 \mathrm{~m}$, the temperatures are warmer than the initial temperature and the profile is stably stratified. The change in temperature relative to the solid profile in Fig. 3a is shown in Fig. 3b. The shapes of these profiles are qualitatively similar to the changes observed during the passage of a storm described in Large and Crawford (1995). In particular, the depth of the transition between regions of warming and cooling varies little during the simulation and the transition region is sharp. The evolution of the currents and temperature in the present simulation are similar to the results obtained by Skyllingstad et al. (2000). However, because of the differences in the 

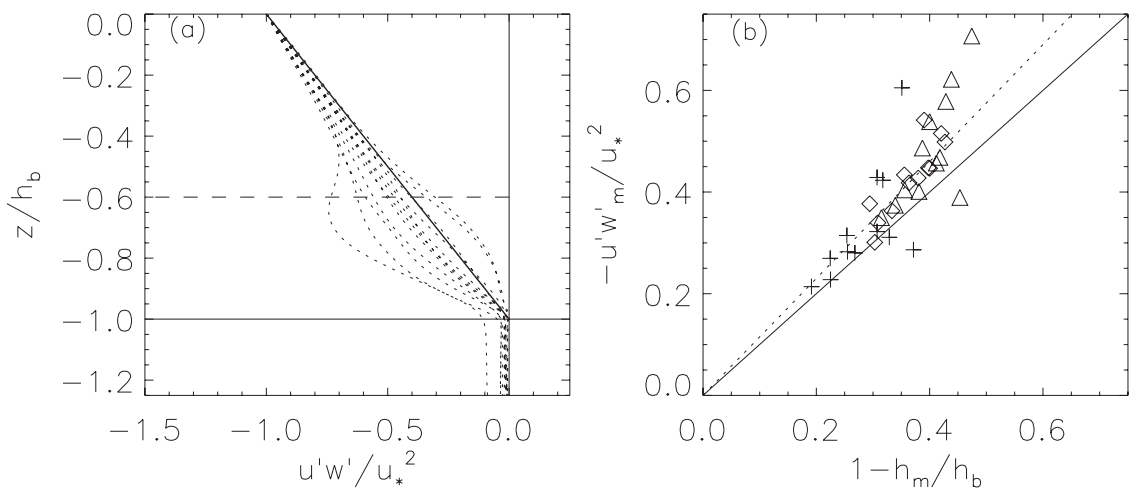

FIG. 2. (a) Plot of the nondimensional stress against $z / h_{b}$. The stress profiles are made nondimensional by $u_{*}^{2}$. The dotted lines are individual profiles from all of the simulations. The dashed line shows the average position of the base of the ML, and the solid line shows $\overline{u^{\prime} w^{\prime}} / u_{*}^{2}=\left(1+z / h_{b}\right)$. (b) Plot of the nondimensional stress at the base of the mixed layer as a function of $\left(1-h_{m} / h_{b}\right)$. Crosses are simulation $\mathrm{A}$, diamonds are simulation $\mathrm{B}$, and triangles are simulation $\mathrm{C}$. The solid line shows the $1: 1$ line, and the dotted line is $y=1.15 x$.

forcing, the magnitudes of the changes seen in the present simulations are much smaller.

The structure of the stratified shear layer that develops below the mixed layer can be described by the vertical gradients of the current velocity and temperature, which are shown in Figs. 4a,b as a function of depth within the stratified shear layer. The gradients have been normalized by the average gradients based on the differences between the mean velocity and temperature in the mixed layer and the velocity and temperature at the base of the stratified layer. The temperature gradient and current shear both increase with depth and have maxima located just above the base of the stratified shear layer. The current shear varies approximately linearly with depth, whereas the temperature gradient profiles have a distinct curvature. Qualitatively, the shape of the temperature gradient profiles is consistent with the observations presented by Large and Crawford (1995) and the LES results described by Skyllingstad et al. (2000). The gradient Richardson number (not shown) is approximately constant over the most of shear layer, with $\mathrm{Ri}_{g} \approx 0.25$.

The average gradients used to normalize the profiles in Figs. 4a,b can be used to define a bulk Richardson number $\mathrm{Ri}_{b}$ as,

$$
\mathrm{Ri}_{b}=\frac{\left(B_{b}-\left\langle B_{m}\right\rangle\right)\left(h_{b}-h_{m}\right)}{\left\langle U_{m}\right\rangle^{2}},
$$

where $B_{b}$ is the mean buoyancy at the base of the stable layer, $\left\langle B_{m}\right\rangle$ is the mean buoyancy in the mixed layer, $\left\langle U_{m}\right\rangle$ is the average mixed layer velocity, and $h_{m}$ is the mixed layer depth.

Figure 5 shows $\mathrm{Ri}_{b}$ as a function of time for the three simulations. In all three simulations, $\mathrm{Ri}_{b}$ initially decreases
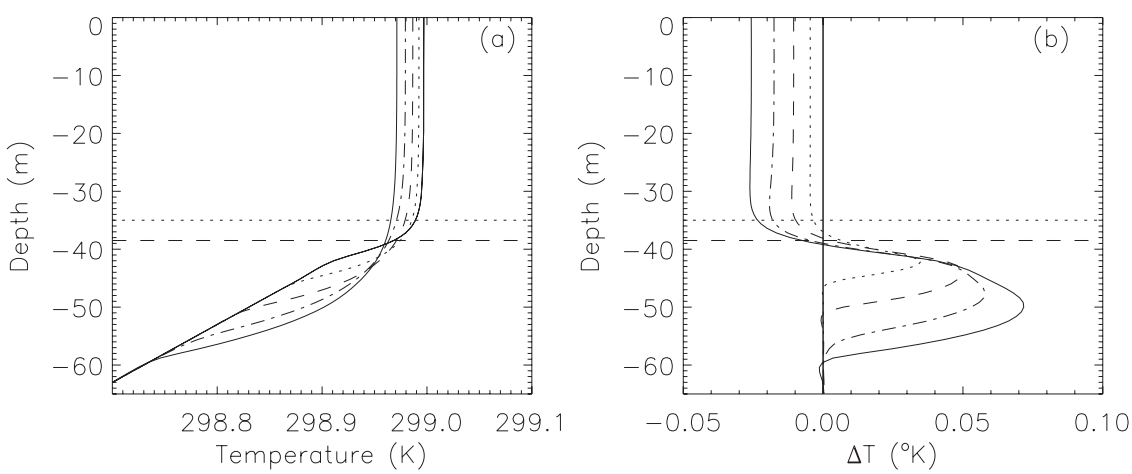

FIG. 3. Evolution with time of (a) temperature profiles and (b) change in temperature relative to the first profile in (a) for simulation A. The horizontal dotted line marks the base of the layer with small vertical gradients, and the horizontal dashed line marks the position of the level at which the temperature change is approximately zero. The line types correspond to profiles at the same times as the profiles in Fig. 1. 

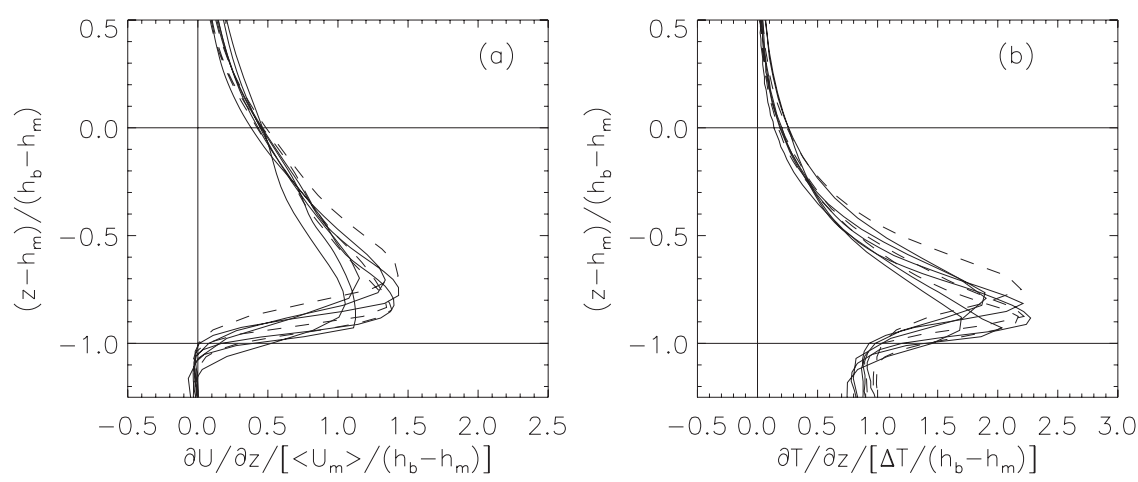

FIG. 4. (a) Profiles of the current shear in the stratified layer. Profiles are normalized by $\left\langle U_{m}\right\rangle /\left(h_{b}-h_{m}\right)$. (b) Profiles of the temperature gradient in the stratified layer. Profiles are normalized by $\Delta T /\left(h_{b}-h_{m}\right)$, where $\Delta T$ is the change in temperature across the stratified layer. Solid curves are simulation A, dashed curves are simulation B, and dashed-dotted-dotteddotted curves are simulation C. Depth is measured relative to the base of the ML and normalized to the depth of the SSL.

with time tending to a constant value at long times. After the initial decrease, the Richardson number tends to a value $0.25-0.3$, which is consistent with the mixing in the stratified shear layer being due to shear-generated turbulence.

Referring to Figs. 1-3, the structure of the boundary layer can be summarized as follows: The stress profile varies smoothly between the surface and the base of the shear layer, defining the OSBL (see Figs. 1a,b). The mean current and temperature profiles (Figs. 1a, 3a,b) show that the boundary layer can be divided into two distinct layers: the mixed layer (ML), in which vertical gradients are small, and below this the stratified shear layer (SSL). For this study, the base of the mixed layer and the top of the stratified shear layer are defined to be the level at which the change in temperature relative to the initial profile is zero.

This picture of the boundary layer derived from the LES results poses the following important questions, which this study will answer:

- What are the turbulent processes responsible for the transports of heat and momentum within the OSBL?

- How is the boundary layer structure maintained?

- How do the exchanges of heat and momentum between the layers relate to the mean structure?

- What controls the rate at which the OSBL deepens?

\section{The turbulent kinetic energy budget}

The TKE budget (Tennekes and Lumley 1972) is fundamental in the analysis of turbulent flows. Including the effects of Stokes shear the TKE budget for quasisteady, horizontally homogeneous conditions can be written as $-\overline{u^{\prime} w^{\prime}} \frac{\partial \bar{U}}{\partial z}-\overline{u^{\prime} w^{\prime}} \frac{\partial u_{s}}{\partial z}+\overline{w^{\prime} b^{\prime}}-\frac{\partial}{\partial z}\left(\overline{w^{\prime} E}+\frac{1}{\rho_{w}} \overline{w^{\prime} p^{\prime}}\right)-\epsilon=0$

where overbars denote spatial and temporal averaging; primes denote fluctuations from the mean; $u, v$, and $w$ are the $x, y$, and $z$ components of the current velocity (where the $x$ axis aligned along the direction of the surface stress and $z$ is vertical); $u_{s}$ is the Stokes drift; $b$ is buoyancy; $E$ is the turbulent kinetic energy = $0.5\left(\overline{u^{\prime 2}}+\overline{v^{\prime 2}}+\overline{w^{\prime 2}}\right) ; p$ is pressure; and $\epsilon$ is the dissipation rate. The first term on the left-hand side of Eq. (2) represents the production of TKE from current shear; the second is production due to the Stokes shear; the third is

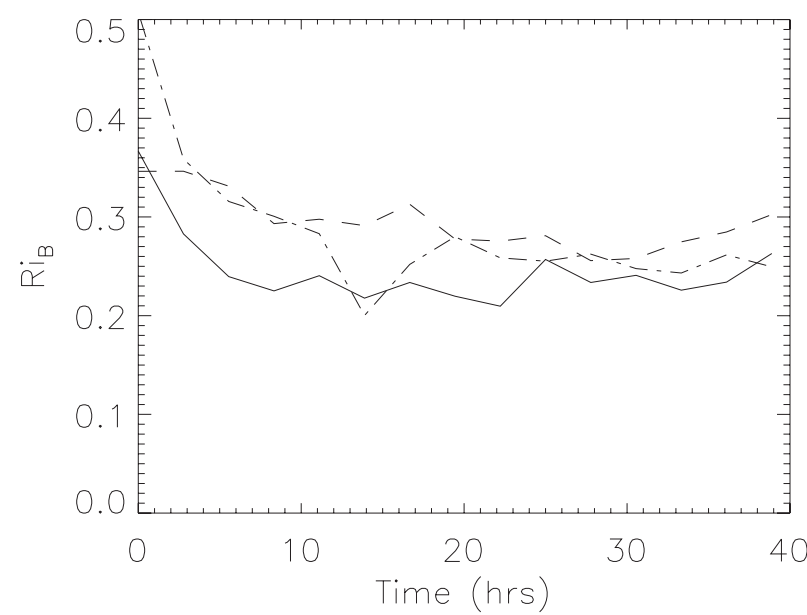

FIG. 5. Plot of the time variation of the bulk Richardson number across the SSL (see text for definition) for all three simulations. Solid curve is simulation $\mathrm{A}$, dashed curve is simulation $\mathrm{B}$, and dashed-dotted curve is simulation C. 

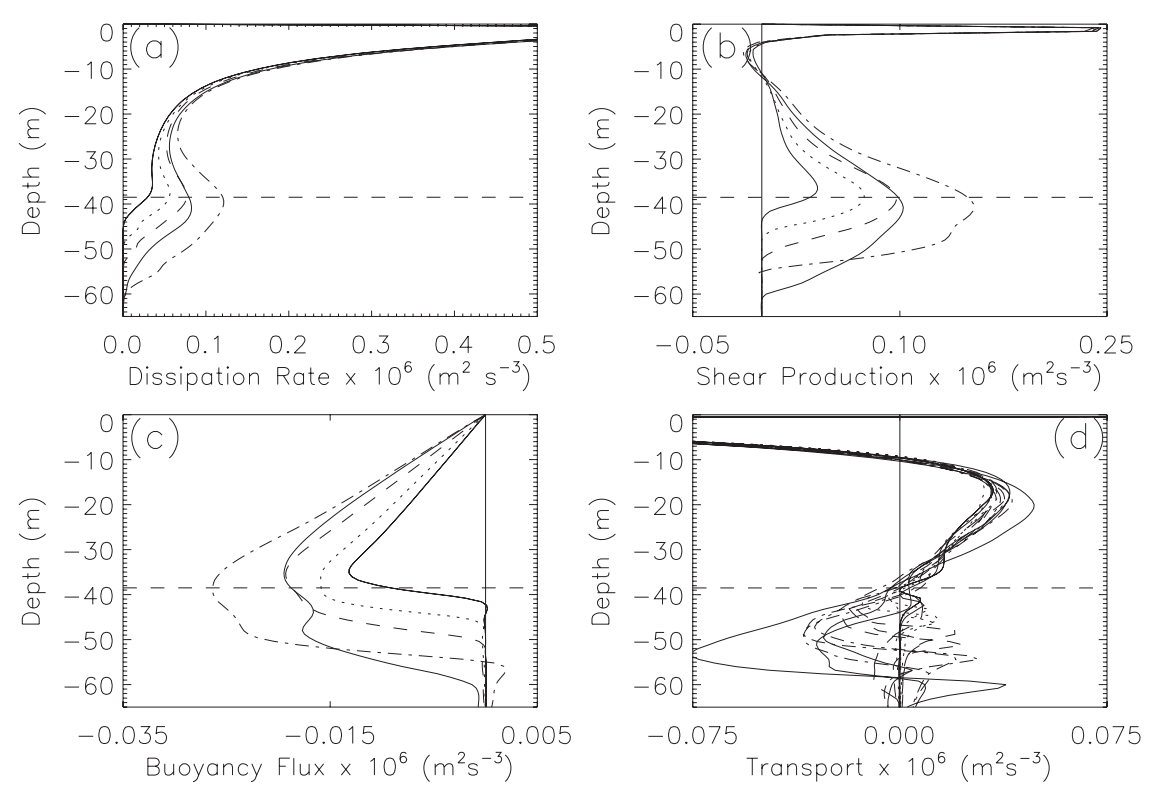

FIG. 6. Evolution, with time, of terms in the TKE budget for simulation A: (a) dissipation rate $\epsilon$; (b) shear production $-\overline{u^{\prime} w^{\prime}} \partial U / \partial z$; (c) buoyancy flux $\overline{w^{\prime} b^{\prime}}$; and (d) transport $-\partial / \partial z\left(\overline{w^{\prime} E}+\overline{w^{\prime} p^{\prime}} / \rho\right)$. In each plot, the solid curve represents the earliest profile with subsequent profiles progressing: dotted, dashed, dashed-dotted, and dashed-dotted-dotteddotted. The horizontal dashed line shows the approximate position of the base of the ML.

the buoyancy term, which in stably stratified conditions is a sink of TKE; and the fourth is the transport term. Unless stated otherwise, in what follows the terms in the TKE budget are shown for the resolved motions only.

Figure 6a shows profiles of the dissipation rate at different times during simulation A. For depths less than about $20 \mathrm{~m}$, the dissipation rate decreases strongly with depth and shows little variation with time. Grant and Belcher (2009) show that the dissipation rate for Langmuir turbulence is $\sim u_{*}^{2} u_{\mathrm{s} 0} / h_{m}$. Because $u_{*}$ and $u_{\mathrm{s} 0}$ are constant and $h_{m}$ varies only slightly with time, the steady dissipation rate above $20 \mathrm{~m}$ is consistent with this region of the boundary layer being dominated by Langmuir turbulence.

One of the features of Langmuir turbulence is that turbulent kinetic energy is transported from the region of Stokes shear into the bulk of the mixed layer (McWilliams et al. 1997; Polton and Belcher 2007; Grant and Belcher 2009). The transport terms for the same times as the dissipation rate profiles are shown in Fig. 6d. The profiles show that TKE is transported from the region of Stokes shear into the bulk of the mixed layer. The forms of the profiles are similar to those presented in Grant and Belcher (2009) and indicate that the Langmuir turbulence is restricted to the mixed layer.

Below $20 \mathrm{~m}$, the dissipation rate increases with increasing depth to reach a local maximum around the base of the mixed layer at $40 \mathrm{~m}$. The magnitude of the dissipation rate at the maximum generally increases with time, although a close examination shows that the variation with time is not necessarily monotonic because of random variability. Profiles of shear production of TKE and the buoyancy flux, corresponding to the dissipation rate profiles, are shown in Figs. 6b,c. Shear production is a maximum at about the same depth as the dissipation rate, with the magnitude increasing with time. The shear production is not restricted to the shear layer and the region around the base of the mixed layer but extends through the full depth of the mixed layer. The buoyancy flux has a minimum at the base of the mixed layer, with the magnitude of the minimum buoyancy flux increasing with time.

The shape of the buoyancy flux profile in Fig. 6c results in the mixed layer cooling and the shear layer warming, as seen in Figs. 3a,b. However, this exchange of heat does not lead to a deepening of the mixed layer as might be expected. A simple model will be developed in section 7 to show how the mixed layer depth is maintained in the face of the buoyancy flux profile in Fig. 6c.

The transport term in the stratified shear layer is variable but on average represents a loss of TKE from the stratified region of the boundary layer. This loss is associated with the production of waves and will be considered further in the discussion section.

The TKE budget shows that the two-layer structure of the boundary layer can be considered to be a consequence 


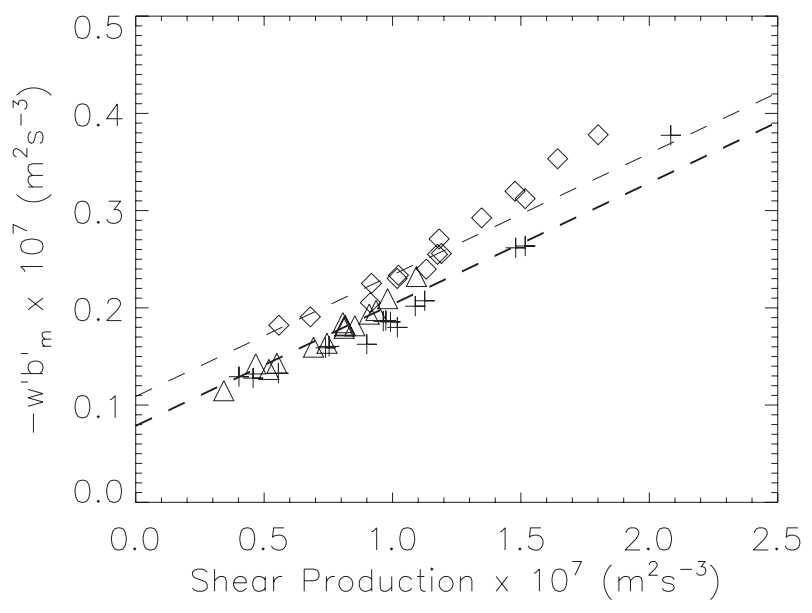

FIG. 7. Plot of the buoyancy flux at the base of the ML as a function of the shear production. The symbols are as follows: crosses for simulation $\mathrm{A}$, diamonds for simulation $\mathrm{B}$, and triangles for simulation $\mathrm{C}$. The dashed lines show the relationship $-\overline{w^{\prime} b_{m}^{\prime}}=-0.11 \overline{u^{\prime} w_{m}^{\prime}} \partial U /\left.\partial z\right|_{m}+0.045 u_{*}^{2} u_{\mathrm{s} 0} / h_{m}$

of there being two distinct turbulent processes in the boundary layer. Langmuir turbulence is restricted to the mixed layer and is responsible for the small gradients in the mixed layer and shear turbulence, which extends through the depth of the boundary layer but is maximal close to the base of the mixed layer.

The shear turbulence is clearly associated with the exchange of heat and momentum between the mixed layer and the shear layer. This is shown in Fig. 7, which compares the magnitude of the buoyancy flux at the base of the mixed layer $\overline{w^{\prime} b_{m}^{\prime}}$ for all three simulations to the magnitude of the shear production. The buoyancy flux increases linearly with increasing shear production but for zero shear production extrapolation implies a nonzero buoyancy flux. This component of the buoyancy flux is associated with the Langmuir turbulence in the mixed layer and should scale with $u_{*}^{2} u_{\mathrm{s} 0} / h_{m}$. (Grant and Belcher 2009). The estimated fluxes for zero shear production are within $\pm 5 \%$ of the entrainment fluxes implied by the results of Grant and Belcher (2009). Although the buoyancy flux associated with the shear production increases with time, the contribution to the buoyancy flux by Langmuir turbulence at the base of the mixed layer remains significant during the simulations. This is consistent with the LES results presented by Skyllingstad et al. (2000), where the cooling of the mixed layer showed some dependence on the specification of the wave forcing.

The production of turbulence by shear increases with time but this is not due to increasing shear but is mainly a result of the increase in $\overline{u^{\prime} w^{\prime}}$ at the base of the mixed layer due to the deepening of the boundary layer (see
Fig. 1b). The shear production at the base of the mixed layer can be estimated as

$$
-\left.\overline{u^{\prime} w^{\prime}} \frac{\partial \bar{U}}{\partial z}\right|_{m} \sim-\overline{u^{\prime} w_{m}^{\prime}} \frac{\left\langle U_{m}\right\rangle}{h_{b}-h_{m}}
$$

where $\rho_{w} \overline{u^{\prime} w_{m}^{\prime}}$ is the turbulent momentum flux at the base of the mixed layer.

Figure $2 \mathrm{~b}$ shows that $\overline{u^{\prime} w_{m}^{\prime}} \propto-u_{*}^{2}\left(1-h_{m} / h_{b}\right)$. Therefore, Eq. (3) can be written as

$$
-\left.\overline{u^{\prime} w^{\prime}} \frac{\partial U}{\partial z}\right|_{m} \sim \frac{u_{*}^{2}\left\langle U_{m}\right\rangle}{h_{b}}
$$

Figures $8 \mathrm{a}, \mathrm{b}$ show the resolved shear production and dissipation at the base of the mixed layer as a function of $u_{*}^{2}\left\langle U_{m}\right\rangle / h_{b}$. The variations in both shear production and dissipation are well correlated with $u_{*}^{2}\left\langle U_{m}\right\rangle / h_{b}$, the shear production $\approx 0.42 u_{*}^{2}\left\langle U_{m}\right\rangle / h_{b}$, and dissipation $\approx 0.32 u_{*}^{2}\left\langle U_{m}\right\rangle / h_{b}$.

Equation (4) is a key result, because it links the rate of generation of shear turbulence in the stratified shear layer directly to the surface forcing. An interpretation of Eq. (4) is that the shear production is proportional to the divergence of the mean kinetic energy flux associated with the action of the Reynolds stresses on the mixed layer velocity (see discussion section). The magnitude of the shear production implies that averaged over the boundary layer about $20 \%$ of the mean kinetic energy flux is converted to turbulence as a result of the shear across the stratified layer.

The link between the surface forcing represented in Eq. (4) and the shear production at the base of the mixed layer is the Reynolds stress profile that spans the boundary layer. This means that the results of this study do not apply to stratified shear turbulence in general but only to the OSBL considered here. However, what is gained from a lack of generality is the possibility of an accurate description of the turbulent processes in the upper ocean boundary layer.

\section{Mixed layer turbulence}

In this section, mixed layer turbulence statistics will be compared with the results of Grant and Belcher (2009). The differences between the simulations in Grant and Belcher (2009) and the present simulations are as follows: the present simulations have the Coriolis parameter set to 0; the initial mixed layer depth is set to $25 \mathrm{~m}$, rather than $33 \mathrm{~m}$ for simulation $\mathrm{B}$; and in simulation $\mathrm{C}$, the stratification below the mixed layer is reduced from 

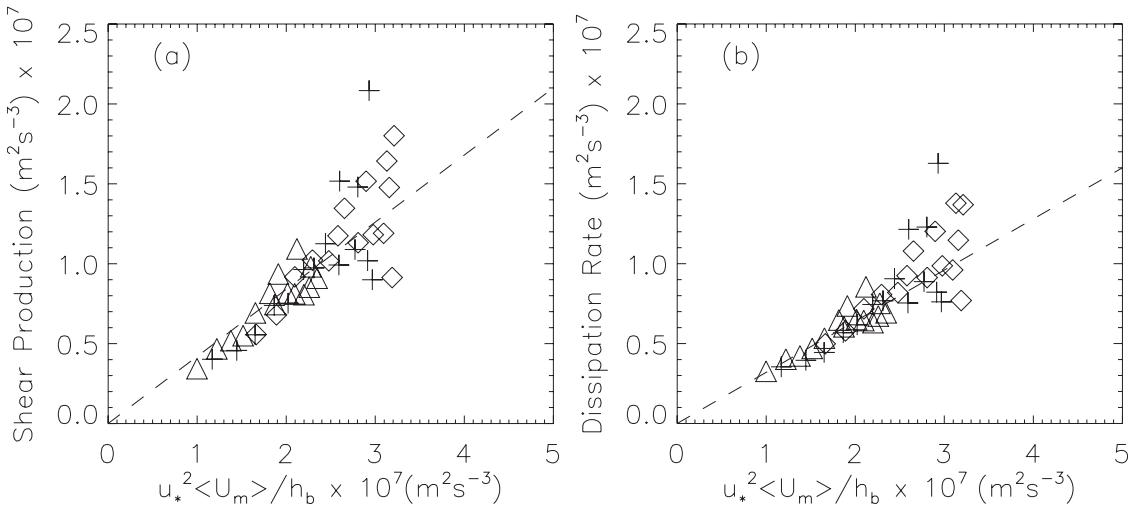

FIG. 8. (a) Shear production at the base of the ML as a function of $u_{*}^{2}\left\langle U_{m}\right\rangle / h_{b}$. The dashed line shows $y=0.42 x$. The symbols are as in Fig. 7b. The dissipation rate at the base of the ML is a function of $u_{*}^{2}\left\langle U_{m}\right\rangle / h_{b}$. The dashed line shows $y=0.32 x$.

0.001 to $0.0005 \mathrm{~K} \mathrm{~m}^{-1}$. In simulation $\mathrm{A}$, the stratification and initial mixed layer depth are the same as those used in Grant and Belcher (2009).

Figure $9 \mathrm{a}-\mathrm{c}$ compare variance profiles scaled by $\left(u_{*}^{2} u_{\mathrm{s} 0}\right)^{2 / 3}$ from the beginning and end of simulation $\mathrm{A}$ with those presented in Grant and Belcher (2009). The profiles from the beginning of simulation $\mathrm{A}$ are similar to those presented in Grant and Belcher (2009), although there are differences. The most obvious difference is that the variance of the longitudinal velocity component $\left(\sigma_{u}^{2}\right)$ is enhanced around the base of the mixed layer because of the shear turbulence. A second difference is that the nondimensional vertical velocity variances are slightly larger in the present simulations than found by Grant and Belcher (2009), which is probably because of the differences in the Coriolis parameter. Grant and Belcher (2009) argue that the Coriolis parameter can influence the production of Langmuir turbulence through the magnitude of the stress gradient at the surface.

Toward the end of simulation $\mathrm{A}, \sigma_{u}^{2}$ increases with depth within the mixed layer and is much larger than that due to Langmuir turbulence. The variance of the lateral velocity component is also enhanced over a large fraction of the depth of the mixed layer, whereas vertical velocity variance shows a small increase close to the base of the mixed layer.

Figures 10a,b compare profiles of the dissipation length scale $\lambda_{\epsilon}=E^{3 / 2} / \epsilon$, scaled with the mixed layer depth, from the present simulations and from Grant and
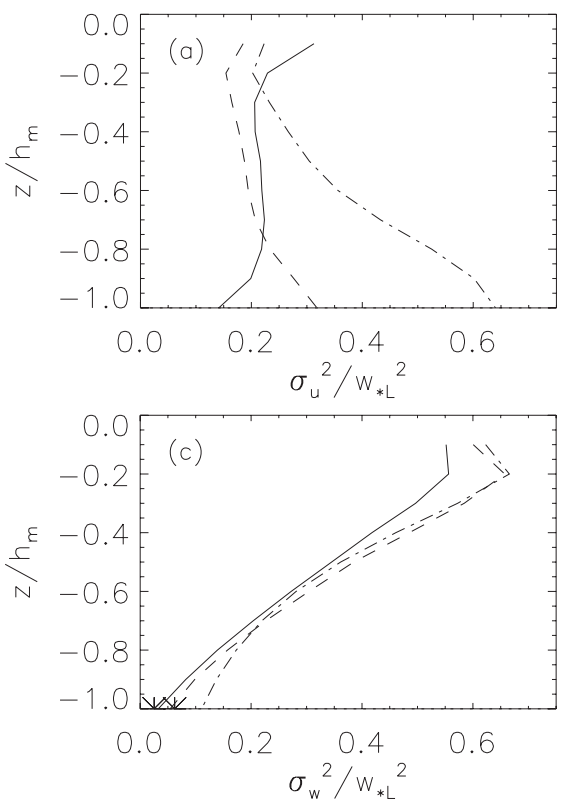

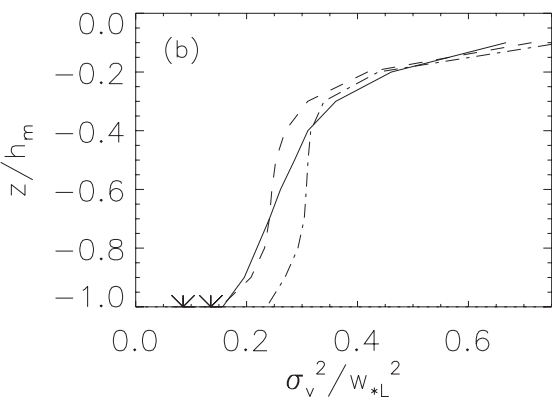

FIG. 9. Variance profiles in the ML normalized by the Langmuir velocity scale $w_{* L}$. Depth is normalized by the ML depth: (a) $\sigma_{u}^{2} / w_{* L}^{2}$, (b) $\sigma_{v}^{2} / w_{* L}^{2}$, and (c) $\sigma_{u}^{2} / w_{* L}^{2}$. The symbols at the base of the ML are the values of the nondimensional variances obtained from Fig. 12. The solid curves are from Grant and Belcher (2009), dashed curves are from the beginning of simulation $\mathrm{A}$, and dotted-dashed curves are from the end of simulation A. 

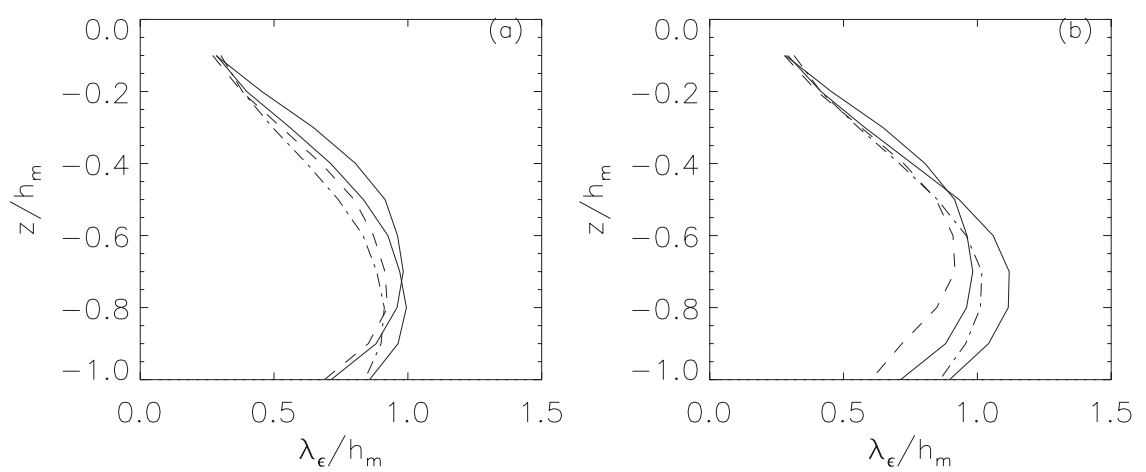

FIG. 10. Profiles of the dissipation length scale normalized by the ML depth: (a) from the start of the simulations and (b) from the end of the simulations. Solid curves are from Grant and Belcher (2009), dashed curves are from simulation A, dashed-dotted curves are from simulation B, and dashed-dotted-dotted-dotted curves are from simulation C.

Belcher (2009). Early in the present simulations (Fig. 10a), the profiles are similar to those presented by Grant and Belcher (2009), although the maximum is slightly deeper in the present simulations. The scaling of the results from the present simulations by the mixed layer depth appears to be reasonable. Figure $10 \mathrm{~b}$ shows that, at the end of the simulations, the shapes of the dissipation length scale profiles are still similar to those in Grant and Belcher (2009). However, although the scaling by mixed layer depth appears reasonable above $z / h=-0.6$, below this there is a significant increase in the variation between the simulations compared to that in Fig. 10a. This is the region of enhanced dissipation because of shear turbulence, and the increased variation in the dissipation length scale suggests that a length scale other than the mixed layer depth is relevant in this region.

The effects of both Langmuir and shear turbulence are important at the base of the mixed layer (see Fig. 7). To account for this in the analysis that follows, a nondimensional parameter $\zeta$ can be formed from the ratio of the production rate of shear turbulence to the production rate of Langmuir turbulence $u_{*}^{2} u_{\mathrm{s} 0} / h_{m}$ (Grant and Belcher 2009): namely,

$$
\zeta=\frac{u_{\mathrm{s} 0} h_{b}}{\left\langle U_{m}\right\rangle h_{m}} .
$$

As constructed, the importance of the shear turbulence increases as $\zeta$ decreases.

The differences between the mixed layer profiles at the start and the end of the simulations can be related to the nondimensional parameter $\zeta$. For the early profiles $\zeta>1$, whereas for the profiles toward the end of the simulations $\zeta<1$. This suggests that $\zeta \approx 1$ marks the boundary between turbulence characteristics at the base of the mixed layer being dominated by Langmuir turbulence $(\zeta>1)$ or by shear turbulence $(\zeta<1)$.

\section{Turbulent velocity and length scale for shear turbulence}

The production of turbulence by current shear at the base of the mixed layer has a significant impact on the turbulence in the mixed layer and the exchanges of heat and momentum across the base of the mixed layer. To understand the characteristics of the shear turbulence further, it is necessary to define relevant velocity and length scales. The dissipation rate for high Reynolds number turbulence can be related to these scales through $\epsilon \sim \nu_{*}^{3} / l_{\epsilon}$, where $\nu_{*}$ is the velocity scale and $l_{\epsilon}$ determines the dissipation length scale $\lambda_{\epsilon}=E^{3 / 2} / \epsilon$ (Tennekes and Lumley 1972). Using Eq. (4) as an estimate of the dissipation rate,

$$
\frac{\nu_{*}^{3}}{l_{\epsilon}} \sim \frac{u_{*}^{2}\left\langle U_{m}\right\rangle}{h_{b}} .
$$

The profiles of the shear stress and the shear production term in the TKE budget suggest that $l_{\epsilon} \sim h_{b}$. To confirm this assumption, Fig. 11 shows the dissipation length scale $\lambda_{\epsilon}$ as a function of $h_{b}$ for $\zeta<1$. The results show $\lambda_{\epsilon} \approx 0.5 h_{b}$, which is consistent with the idea that the shear-driven turbulence is a boundary layer phenomenon. The dependence of the dissipation length scale on the boundary layer depth also explains the variation in $\lambda_{\epsilon} / h_{m}$ in the lower part of the mixed layer in Fig. $10 \mathrm{~b}$.

Using the result for the dissipation length scale in Eq. (6), the velocity scale for the shear-generated turbulence $\nu_{*}$ is

$$
\nu_{*}=\left(u_{*}^{2}\left\langle U_{m}\right\rangle\right)^{1 / 3}
$$

To test the proposed velocity scale, we consider the variances of the velocity components at the base of the mixed layer. A similarity hypothesis that accounts for the effects of both Langmuir and shear turbulence is 


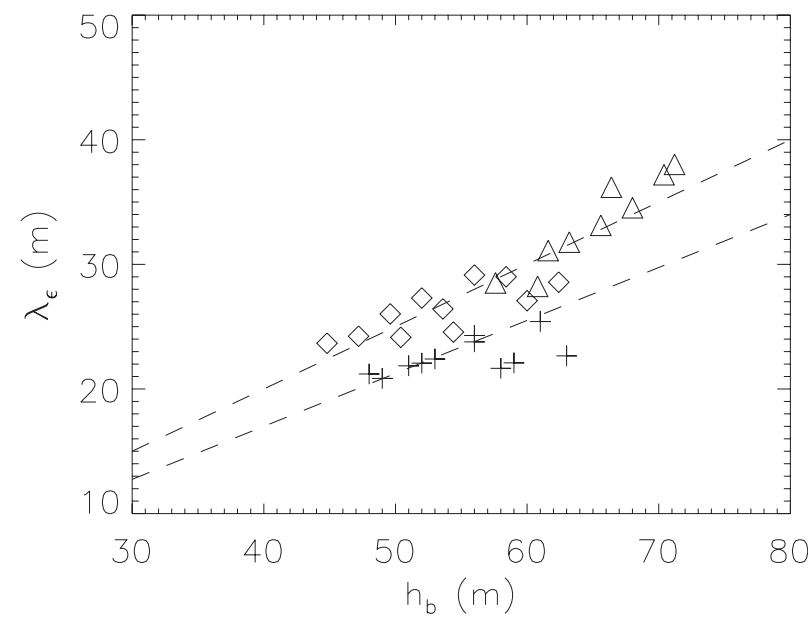

FIG. 11. Plot of the dissipation length scale, for $\zeta<1$, against the depth of the boundary layer $h_{b}$. Symbols are as in Fig. 7 .

$$
\frac{\sigma_{i}^{2}}{\nu_{*}^{2}}=f_{i}(\zeta)
$$

where $\sigma_{i}^{2}$ represents the velocity component variances, with $i=(u, v, w)$, and $f_{i}(\zeta)$ represents similarity functions for the variances. The similarity hypothesis is not unique, because the velocity scale for Langmuir turbulence $w_{* L}$ could be used in place in of place of $\nu_{*}$. However, in this study we are interested in the shear component of the turbulence and so it is sensible to choose $\nu_{*}$ as the primary velocity scale. The similarity functions $f_{i}(\zeta)$ need to be determined, which can be done empirically using simple statistical fits to the LES results. It is, however, useful to consider whether physical arguments can be used to constrain the form of the functions $f_{i}(\zeta)$.

From the estimates for the production of turbulence by Stokes shear and current shear, the TKE budget at the base of the mixed layer can be written in terms of scales as

$$
A \frac{u_{*}^{2} U_{m}}{h_{b}}+C \frac{u_{*}^{2} u_{\mathrm{s} 0}}{h_{m}} \sim \frac{w_{* G}^{3}}{l_{\epsilon}},
$$

where $A$ and $C$ are coefficients relating the scales to the magnitudes of the actual production terms in the TKE budget. The right-hand side of Eq. (9) defines a generalized velocity scale $w_{* G}$ that is intended to describe the combination of Langmuir and shear turbulence. Unlike the velocity scales for the individual processes, the generalized scale is not unique but depends on the quantity being considered through the coefficients $A$ and $C$.

A complication with defining $w_{* G}$ in this way is that the Langmuir and shear turbulence are associated with different length scales, which raises the question as to which of these length scales should be used on the right-hand side of Eq. (9). Given that we are interested primarily in situations where the shear contribution to the turbulence at the base of the mixed layer dominates over the contribution from Langmuir turbulence, it seems reasonable to take the length scale to be $\sim h_{b}$, which gives

$$
\omega_{* G}^{2}=\nu_{*}^{2}(A+C \zeta)^{2 / 3}
$$

Assuming that nondimensional velocity variances scaled with $\omega_{*_{G}}^{2}$ are constant for small values of $\zeta$, then a reasonable assumption for the form of $f_{i}$ in Eq. (8) is $f_{i}(\zeta)=\left(A_{i}+C_{i} \zeta\right)^{2 / 3}$, where $A_{i}^{2 / 3}$ is the nondimensional variance $\sigma_{i}^{2} / \nu_{*}^{2}$ for the shear component of the turbulence at the base of the mixed layer and $C_{i}^{2 / 3}$ is the nondimensional variance for Langmuir turbulence, $\sigma_{i}^{2} / w_{* L}^{2}$. Note that, as $\zeta \rightarrow 0, w_{* G} \rightarrow w_{* L}$.

Figure 12a shows $\sigma_{u}^{2}$ as a function of $\nu_{*}^{2}$. The variance appears to be proportional to $\nu_{*}^{2}$ with $\sigma_{u}^{2} / \nu_{*}^{2} \approx 0.35$. This is supported by Fig. 12b, which shows $\sigma_{u}^{2} / \nu_{*}^{2}$ to be independent of $\zeta$. The reason for this simple proportionality between $\sigma_{u}^{2}$ and $\nu_{*}^{2}$ is that the contribution to $\sigma_{u}^{2}$ from shear turbulence at the base of the mixed layer is significantly larger than that due to Langmuir turbulence throughout the simulation (see Fig. 9a).

Figures 12c,d show $\sigma_{v}^{2} / \nu_{*}^{2}$ and $\sigma_{w}^{2} / \nu_{*}^{2}$ as functions of $\zeta$. The assumed form of $f_{i}$ appears to provide a reasonable fit to these results. However, the variances from simulation $\mathrm{A}$ are generally smaller than those from simulations $\mathrm{B}$ and $\mathrm{C}$, which suggests that one or both of the coefficients $A_{i}$ and $C_{i}$ for simulation A should differ from the coefficients from simulations B and C. Any such differences should be related to differences in a nondimensional parameter, and in this case the relevant parameter appears to be an interfacial Richardson number defined as

$$
\mathrm{Ri}_{i}=\frac{\Delta B h_{m}^{2}}{\left(h_{b}-h_{m}\right) w_{* L}^{2}} .
$$

The interfacial Richardson number measures the ratio of the kinetic energy in the Langmuir turbulence to the work needed to perturb the stratified layer over a distance $\propto h_{m}$. This is analogous to the Richardson number used to characterize entrainment in convective mixed layers (Sullivan et al. 1998), with $\Delta B h_{m} /\left(h_{b}-h_{m}\right)$ playing the role of the buoyancy jump at the base of the mixed layer.

The values of $\mathrm{Ri}_{i}$ for simulations $\mathrm{A}, \mathrm{B}$, and $\mathrm{C}$ are 135 , 67 , and 57 , and by this measure the stratification for simulation $\mathrm{A}$ is greater than that for simulations $\mathrm{B}$ and C. Because $\mathrm{Ri}_{i}$ relates to the effect that stratification has on the Langmuir component of the turbulence (through $w_{* L}$ ) the variations in $\mathrm{Ri}_{i}$ suggests that the differences in 

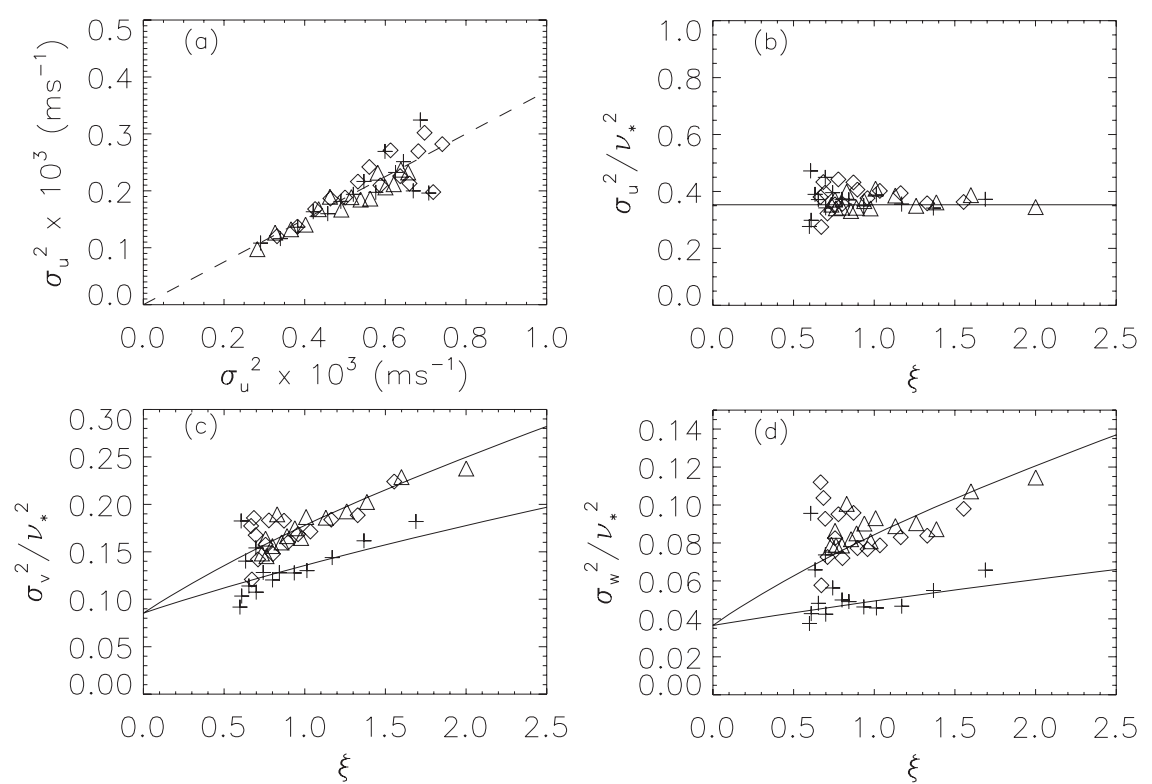

FIG. 12. (a) Plot of $\sigma_{u}^{2}$ against $\nu_{*}^{2}=\left(u_{*}^{2}\left\langle U_{m}\right\rangle\right)^{2 / 3}$. The dashed line shows $y=0.35 x$. (b) Plot of the nondimensional variance $\sigma_{u}^{2} / \nu_{*}^{2}$ against $\zeta$. The solid line shows $\sigma_{u}^{2} / \nu_{*}^{2}=0.35$. (c) As in (b), but for $\sigma_{u}^{2} / \nu_{*}^{2}$. The curves show $\sigma_{v}^{2} / \nu_{*}^{2}=(0.025+0.05 \zeta)^{2 / 3}$ (upper curve) and $\sigma_{u}^{2} / \nu_{*}^{2}=$ $(0.025+0.025 \zeta)^{2 / 3}$ (lower curve). (d) As in (b), but for $\sigma_{w}^{2} / \nu_{*}^{2}$. The curves show $\sigma_{w}^{2} / \nu_{*}^{2}=$ $(0.025+0.0155 \zeta)^{2 / 3}$ (upper curve) and $\sigma_{w}^{2} / \nu_{*}^{2}=(0.007+0.004 \zeta)^{2 / 3}$ (lower curve). Symbols in all plots are as in Fig. 7.

the nondimensional variances seen in Figs. $12 \mathrm{c}$,d should be associated with the coefficients $C_{\nu}$ and $C_{w}$. The curves in Figs. 12c,d have therefore been fitted to LES results by assuming that the coefficients $A_{v}$ and $A_{w}$ are the same for all of the simulations. The nondimensional variances, $\sigma_{u}^{2} / \nu_{*}^{2}, \sigma_{v}^{2} / \nu_{*}^{2}$, and $\sigma_{w}^{2} / \nu_{*}^{2}$ for the shear turbulence $(\zeta=0)$ obtained in this way are $0.34,0.086$, and 0.033 .

The ratios $\sigma_{v}^{2} / \sigma_{u}^{2}$ and $\sigma_{w}^{2} / \sigma_{v}^{2}$ are 0.23 and 0.38 , which differ from those found in conventional boundary layer shear flows. Grant (1992), for example, found $\sigma_{v}^{2} / \sigma_{u}^{2}=$ 0.52 and $\sigma_{w}^{2} / \sigma_{v}^{2}=0.5$ for the lower part of the neutral atmospheric boundary layer. Typical values in the atmospheric surface layer are $\sigma_{v}^{2} / \sigma_{u}^{2}=0.64$ and $\sigma_{w}^{2} / \sigma_{v}^{2}=$ 0.42 (Panofsky and Dutton 1984). For the present flow, it appears that relatively more energy resides in the longitudinal velocity component than in the lateral components when compared to a more usual boundary layer shear flow.

The values of $C_{v}$ and $C_{w}$ obtained for simulation A are 0.19 and 0.025 , and for simulations $B$ and $C$ the values are 0.13 and 0.067 . These are comparable to the magnitudes of $\sigma_{v}^{2} / w_{*_{L}}^{2}$ and $\sigma_{w}^{2} / w_{*_{G}}^{2}$ for Langmuir turbulence at the base of the mixed layer in Figs. 9b,c.

\section{Exchanges across the base of the mixed layer}

Figure 7 shows that the shear component of the turbulence leads to the exchange of heat and presumably momentum between the mixed layer and the stratified shear layer. In resonantly driven mixing, the exchange of heat between the mixed layer and the stratified shear layer can be large (Large and Crawford 1995). Entrainment parameterizations based on the assumption that the transition region between the mixed layer and the nonturbulent thermocline has negligible thickness are clearly not valid in the present case. Since the base of the mixed layer is marked by significant production of turbulence, the use of an eddy diffusivity to parameterize exchanges would seem to be more appropriate.

The diffusivity for momentum is defined as

$$
K_{M}=-\frac{\overline{u^{\prime} w_{m}^{\prime}}}{\partial U /\left.\partial z\right|_{m}},
$$

and that for heat is defined as

$$
K_{H}=-\frac{\overline{w^{\prime} T_{m}^{\prime}}}{\partial T /\left.\partial z\right|_{m}},
$$

The fluxes used to estimate the eddy diffusivity from Eqs. (12) and (13) include the subgrid contributions. The subgrid contribution to the total fluxes at the base of the mixed layer are between $10 \%$ and $25 \%$, with the subgrid 

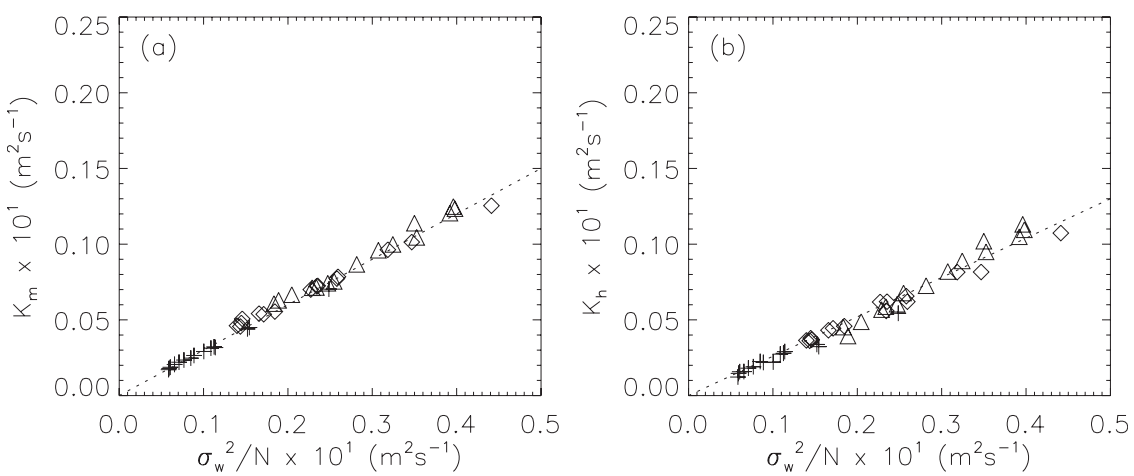

FIG. 13. Diffusivities for momentum and heat as functions of $\sigma_{w}^{2} / N$. (a) Diffusivity for momentum. Dotted line is $y=0.35 x$. (b) Diffusivity for heat. Dotted line shows $y=0.26 x$. Symbols are as in Fig. 7.

contribution being greatest for simulation $\mathrm{A}$, which also has the highest value of $R i_{i}$.

The base of the mixed layer lies within the stratified layer (see Fig. 4). For stratified turbulence, Pearson et al. (1983) suggest that $K \sim \sigma_{w}^{2} / N$, where $N$ is the BruntVäisälä frequency. Figures 13a,b show $K_{M}$ and $K_{H}$ at the base of the mixed layer diagnosed from the LES as functions of $\sigma_{w}^{2} / N$. It is clear that, for both momentum and heat, $K \propto \sigma_{w}^{2} / N$ is a good approximation, with $K_{M}=0.25 \sigma_{w}^{2} / N$ and $K_{H}=0.25 \sigma_{w}^{2} / N$. The values of $K_{M} N / \sigma_{w}^{2}$ and $K_{H} N / \sigma_{w}^{2}$ are comparable to those obtained for other stratified shear flows. Nieuwstadt (1984) and Hunt et al. (1985) found $K_{H} N / \sigma_{w}^{2} \sim 0.17-0.25$ in the stable atmospheric boundary layer. Otte and Wyngaard (2001) found $K_{H} N / \sigma_{w}^{2}=0.26$ and $K_{M} N / \sigma_{w}^{2}=0.35$ from LES of the stable interfacial layer in shear-driven and convective atmospheric boundary layers.

Figures 13a,b are local similarity results: that is, the scaling is based on local mean and turbulence quantities (Nieuwstadt 1984). Although local similarity highlights the common features of stratified turbulence, the parameterization of fluxes in a large-scale model requires that the diffusivities be related to the basic scales that determine the structure of the boundary layer.

The local parameterization of the eddy diffusivity using $\sigma_{w}^{2} / N$ can be written in terms of scales as

$$
K_{*} \sim \nu_{*}\left(A_{w}+C_{w} \zeta\right)^{1 / 3} l_{b},
$$

where $l_{b}=\sigma_{w} / N$ is the buoyancy length scale (Otte and Wyngaard 2001). Figure 14 shows that the buoyancy length scale is proportional to the boundary layer depth, with $l_{b}=A_{l_{b}} h_{b}$, where the coefficient $A_{l_{b}}$ appears to be a function of the interfacial Richardson number. There appears to be a similar dependency on $\mathrm{Ri}_{i}$ in the dissipation length scale $\lambda_{\epsilon}$ (see Fig. 11).
The final scaling for eddy diffusivities can be written as

$$
K_{*}=A_{l_{b}}\left(A_{w}+C_{w} \zeta\right)^{1 / 3} \nu_{*} h_{b} .
$$

Using values for the coefficients $A_{l}, A_{w}$, and $C_{w}$ obtained from the LES results, Figs. $15 \mathrm{a}, \mathrm{b}$ show the diffusivities of momentum and heat as functions of $K_{*}$. The scaling works well, giving $K_{M}=0.35 K_{*}$ and $K_{H}=0.3 K_{*}$.

\section{A bulk boundary layer model}

In the previous sections, the analysis of the LES results have been used to provide a detailed picture of the structure of the boundary layer and the turbulence associated with shear at the base of the mixed layer. This description of the mean and turbulence structure of the boundary layer assumes that the mixed layer and boundary layer depths, which provide the basic turbulence length scales, can be determined. In this section, the evolution of the depths of the mixed layer and boundary layer will be considered.

A useful framework for considering this problem is provided by bulk boundary layer models, which make assumptions about the mean structure of the boundary layer. Mixed layer models are a simple form of bulk model, in which mean gradients in the boundary layer are assumed to be zero and the interface between the turbulent boundary layer and the nonturbulent interior is assumed to have negligible depth and is marked by jumps in buoyancy and velocity (Kraus and Turner 1967; Garwood 1977). The present boundary layer is more complex. There are two layers, the mixed layer and the stratified shear layer. In the stratified shear layer, gradients are not small and there do not appear to be any jumps in the mean buoyancy or current either at the base of the mixed layer or at the base of the boundary layer. 


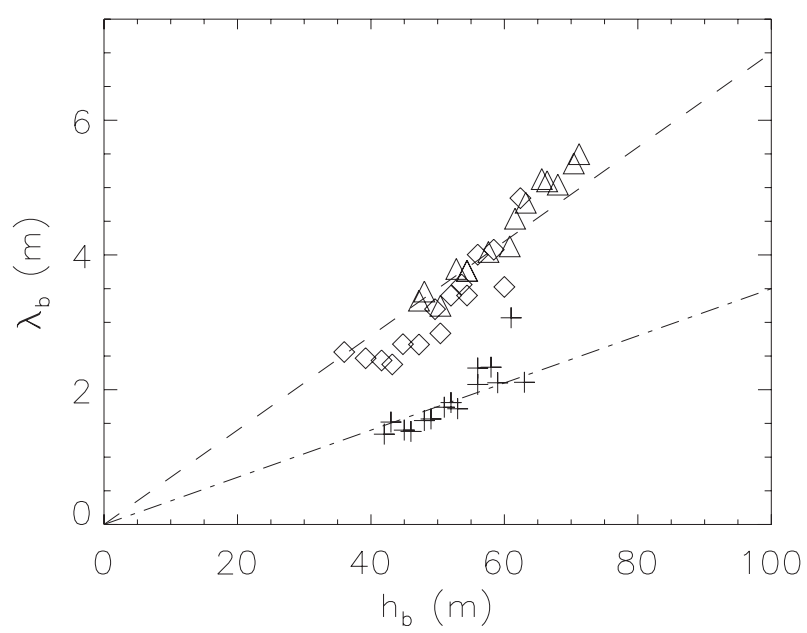

FIG. 14. The buoyancy length scale $\lambda_{b}=\sigma_{w} / N$ at the base of the $\mathrm{ML}$ as a function of the boundary layer depth. Symbols are as in Fig. 7.

Although there are no jumps in mean buoyancy and velocity at the base of the boundary layer, Figs. 4a,b show that the vertical gradients of temperature and current velocity change rapidly with depth in this region. This suggests that it may be useful to describe the mean structure of the boundary layer in terms of the vertical gradients of the mean temperature and velocity. Figures $16 a, b$ show the mean structure of the boundary layer assumed in the bulk model, which will be used to determine the time variation of the boundary layer and mixed layer depths. The rapid changes in the gradients of temperature and velocity at the base of the boundary layer have been idealized to jumps. The gradients around the base of the mixed layer are continuous, but the distance they extend into the mixed layer is proportional to the thickness of the stratified shear layer. This interface region is also idealized as an infinitesimally thick layer with jumps in the gradients. The turbulent flux profiles assumed in the model are shown in Figs. 16c,d. The stress profile is almost linear, and like the mean gradients the stress gradient at the base of the boundary layer is assumed to decrease to zero over an infinitesimally thin layer. The buoyancy flux is assumed to be a minimum at the base of the mixed layer.

Although the present model is based on gradients, it is not fundamentally different from conventional mixed layer models. In a mixed layer model, the assumption that the depth of the stratified layer is much smaller than the depth of the well mixed layer leads to discontinuities being introduced in the mean current and buoyancy profiles as well as the turbulent flux profiles at the base of the mixed layer. Because the depth of the stratified layer is assumed to be infinitesimal, its internal structure does not have to be defined. In contrast, for the boundary layer considered here the structure of the stratified shear layer has to be defined and the use of gradients makes sense because these have a direct link to the turbulent fluxes.

\section{a. The depth of the boundary layer}

For horizontally homogeneous conditions, the equation for the mean current shear is

$$
\frac{\partial}{\partial t} \frac{\partial U}{\partial z}=-\frac{\partial^{2} \overline{u^{\prime} w^{\prime}}}{\partial z^{2}} .
$$

Integrating Eq. (16) from just below to just above the base of the boundary layer to just above and taking the limit $h_{b+} \rightarrow h_{b-}$ gives

$$
\left.\frac{\partial U}{\partial z}\right|_{h_{b+}} \frac{\partial h_{b}}{\partial t}=-\left.\frac{\partial \overline{u^{\prime} w^{\prime}}}{\partial z}\right|_{h_{b+}},
$$

From Fig. 4b, the current shear just above the base of the boundary layer is $\approx\left\langle U_{m}\right\rangle /\left(h_{b}-h_{m}\right)$, whereas the stress divergence at the base of the boundary layer is
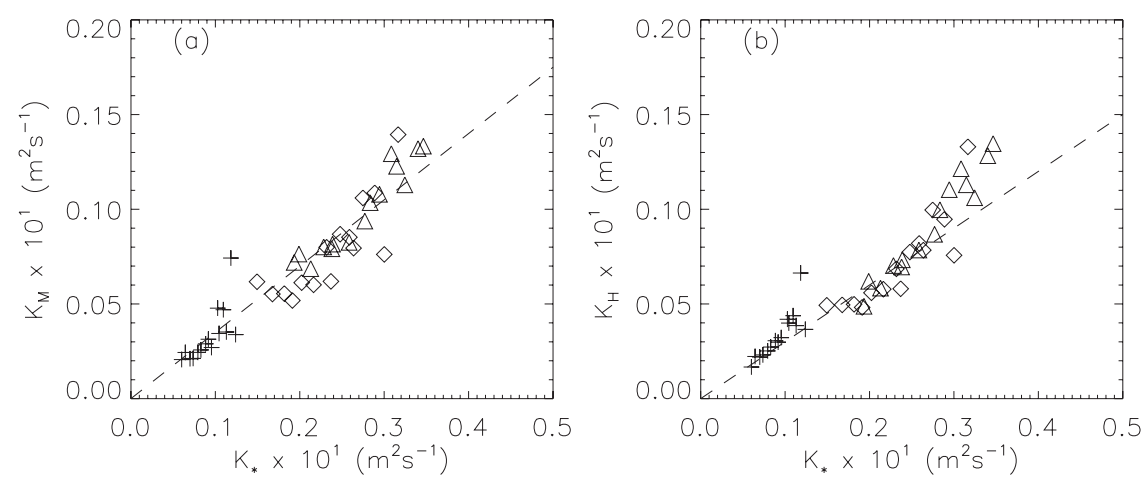

FIG. 15. Diffusivities for momentum and heat as functions of $K_{*}$. (a) Diffusivity for momentum. Dotted line is $y=0.35 x$. (b) Diffusivity for heat. Dotted line shows $y=0.3 x$. Symbols are as in Fig. 7. 

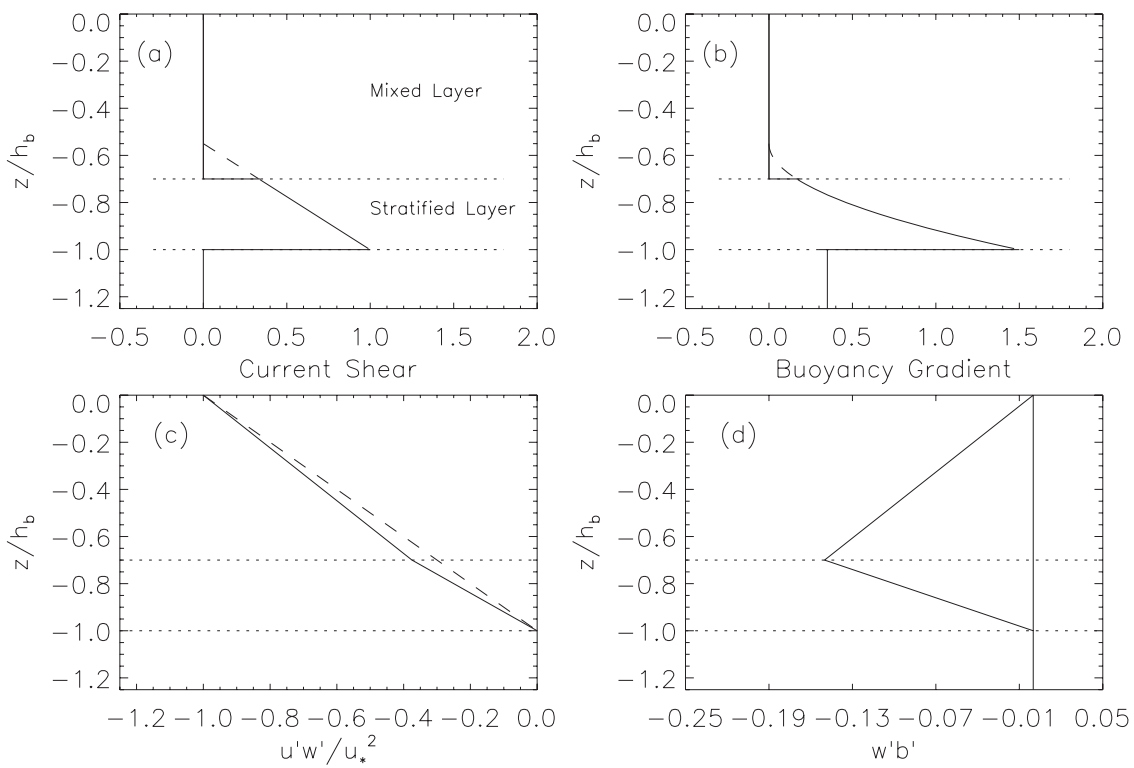

FIG. 16. Schematic of the boundary layer structure assumed by the bulk model described in the text. (a) Profile of the current shear. The solid curves are the assumed profiles, and the dashed section illustrates the actual profile above the base of the ML. (b) As in (a), but for the temperature gradient. (c) Profile of the nondimensional momentum flux (solid line). The dashed line shows a linear profile. (d) Profile of the buoyancy flux. In all plots, the horizontal dotted lines mark the base of the ML and the base of the boundary layer.

$\partial \overline{u^{\prime} w^{\prime}} /\left.\partial z\right|_{h} \sim-u_{*}^{2} / h_{b}$. Using these approximations in Eq. (17), the equation for the depth of the boundary layer can be written as

$$
\frac{\partial h_{b}}{\partial t}=A_{b}\left(1-\frac{h_{m}}{h_{b}}\right) \frac{u_{*}^{2}}{\left\langle U_{m}\right\rangle},
$$

where $A_{b}$ is a constant $\approx 1$. Note that Eq. (18) only applies to the deepening boundary layer. A more general model would be needed to describe situations in which the boundary layer shoals, as might occur with nonsteady forcing.

Figures $17 \mathrm{a}-\mathrm{c}$ compare the boundary layer depth calculated from Eq. (18) with $h_{b}, h_{m}$, and $\left\langle U_{m}\right\rangle$ taken from the LES. The boundary layer depths obtained by integrating Eq. (18) are compared with those from the LES, because vertical resolution and discretization errors in the LES diagnostics make estimates of $\partial h_{b} / \partial t$ inaccurate over the averaging time for turbulence statistics. For each simulation, the initial depth has also been taken from the LES.

The agreement between $h_{b}$ calculated from integrating Eq. (18) and the boundary layer depth obtained from the LES is good. Over the length of the simulation, the average $\partial h_{b} / \partial t$ from the LES agrees well with Eq. (18)
A feature of Eq. (18) is that the deepening of the boundary layer does not depend on the stratification of the thermocline. This is supported by the LES results in that the rate of deepening of the boundary layer is similar for simulations $\mathrm{A}$ and $\mathrm{C}$, which differ in the thermocline stratification. However, the boundary layer depth for simulation $\mathrm{C}$ (with weaker stratification) is greater than in simulation $\mathrm{A}$ at the same time, suggesting that stratification has some effect on the initial evolution of the boundary layer.

The argument leading to Eq. (18) could also be applied to the buoyancy, because this also has jumps in both the gradient and flux divergence at the base of the boundary layer. The resulting equation is

$$
\frac{\partial h_{b}}{\partial t}=-A_{\mathrm{wb}} \frac{\overline{w^{\prime} b_{m}^{\prime}}}{\left(h_{b}-h_{m}\right)} \frac{1}{\left[\gamma \Delta B /\left(h_{b}-h_{m}\right)-\Gamma_{\mathrm{ext}}\right]},
$$

where $\gamma \Delta B /\left(h_{b}-h_{m}\right)$ is the buoyancy gradient just above the base of the boundary layer, $\Gamma_{\text {ext }}$ is the buoyancy gradient in the thermocline, and $A_{\mathrm{wb}}$ is a constant.

Equation (19) is as valid as Eq. (18) for describing the deepening of the boundary layer, although Eq. (18) is consistent with the boundary layer being shear driven. However, $\partial h_{b} / \partial t$ from Eq. (19) must give the same rate of increase in boundary layer depth. Equating the 

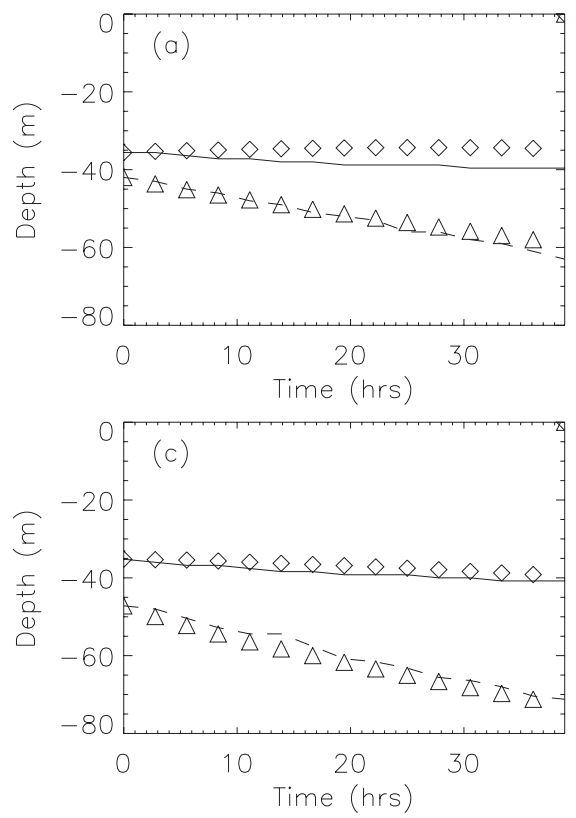

right-hand sides of Eqs. (18) and (19) and recalling that $\overline{w^{\prime} b_{m}^{\prime}} \approx(0.04+0.045 \zeta) u_{*}^{2}\left\langle U_{m}\right\rangle / h_{b}$ gives

$$
\mathrm{Ri}_{b}=A_{\mathrm{Ri}} \frac{(0.04+0.045 \zeta)}{\left[\gamma-\Gamma_{\mathrm{ext}}\left(h_{b}-h_{m}\right) / \Delta B\right]},
$$

where $A_{\mathrm{Ri}}$ is a constant.

Equation (20) implies that the bulk Richardson number is not strictly constant but is a function of $\zeta$ and $\Gamma_{\text {ext }}\left(h_{b}-h_{m}\right) / \Delta B$. Figure 18 compares the bulk Richardson number diagnosed from the LES with those calculated from Eq. (20). Although the variation in $\mathrm{R} i_{b}$ is not large, the LES values are consistent with Eq. (20) with $A_{\mathrm{Ri}}=3.5$.

The dependence of $\mathrm{Ri}_{b}$ on the nondimensional parameter $\zeta$ shows that the structure of the stratified layer depends to some extent on the mixed layer turbulence. The variation in $\mathrm{Ri}_{b}$ shown in Fig. 5 is similar to the time variation in $\zeta$. Equation (20) shows that the bulk Richardson number also depends on the stratification of the thermocline $\Gamma_{\text {ext }}$. However, in these simulations, $\Gamma_{\text {ext }}\left(h_{b}-h_{m}\right) / \Delta B \approx 1$. This is because the change in $\Delta B$ is dominated by the change in the mean buoyancy at the base of the boundary layer as the boundary layer depth decreases. The change due to the decrease in the mixed layer buoyancy is much smaller.

\section{b. The depth of the mixed layer}

Equation (20) shows that the bulk Richardson number is not strictly constant. However, the variations shown in Figs. 5 and 18 are relatively small. Differentiating

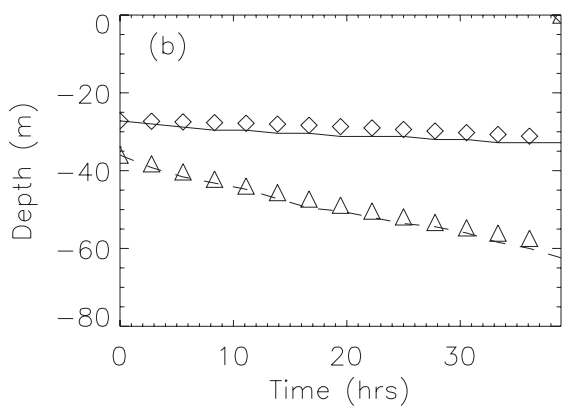

FIG. 17. Comparison between ML depths calculated from Eq. (26) and boundary layer depth calculated from Eq. (18) and with $h_{m}$ and $h_{b}$ diagnosed from the LES. Lines show LES results: solid lines are ML depth and dashed lines are boundary layer depth. The symbols show calculated depths: diamonds are ML depth and triangles are boundary layer depth. Results are for (a) simulation A, (b) simulation $\mathrm{B}$, and (c) simulation $\mathrm{C}$.

Eq. (1) with respect to time and assuming that the time rate of change of $\mathrm{Ri}_{b}$ is small gives

$$
-\mathrm{Ri}_{\mathrm{cr}} \frac{\partial\left\langle U_{m}\right\rangle^{2}}{\partial t}+\left(h_{b}-h_{m}\right) \frac{\partial \Delta B}{\partial t}+\Delta B \frac{\partial\left(h_{b}-h_{m}\right)}{\partial t}=0,
$$

where $\mathrm{Ri}_{\text {cr }}$ is the constant value taken by the bulk Richardson number. Given equations for $\partial\left\langle U_{m}\right\rangle^{2} / \partial t$ and $\partial \Delta B / \partial t$, Eq. (21) provides an equation for the thickness of the stratified shear layer that ensures that the Richardson number remains constant.

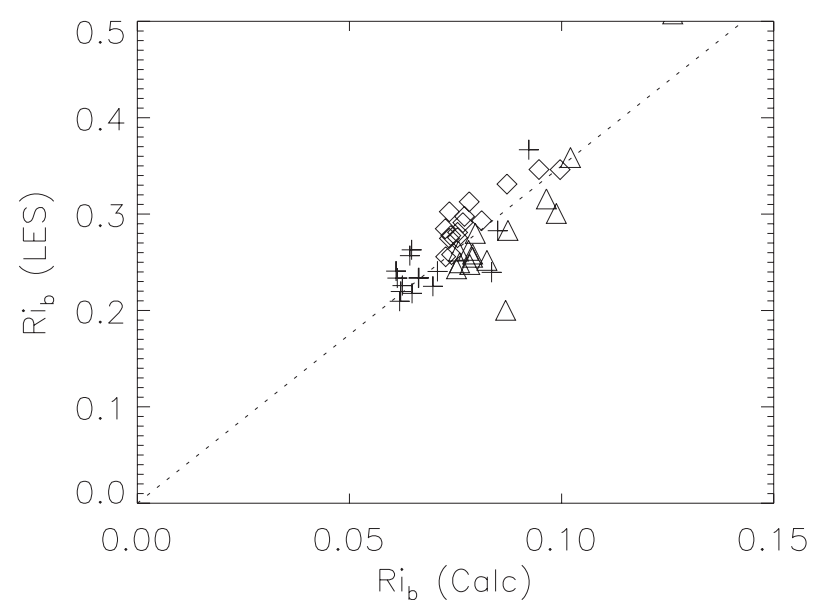

FIG. 18. Comparison between bulk Richardson numbers diagnosed from the LES and calculated from Eq. (20). The crosses are simulation $\mathrm{A}$, diamonds are simulation $\mathrm{B}$, and triangles are simulation $\mathrm{C}$. The dotted line shows $y=3.5 x$. 
The equation governing $\Delta B$ can be obtained by integrating the equation for the buoyancy gradient over the depth of the stratified shear layer, so

$$
\int_{h_{b}^{-}}^{h_{m}^{+}} \frac{\partial \Gamma}{\partial t} d z=-\int_{h_{b}^{-}}^{h_{m}^{+}} \frac{\partial^{2} \overline{w^{\prime} b^{\prime}}}{\partial z^{2}}
$$

where $\Gamma=\partial B / \partial z$. The limits of integration are taken from just below the base of the boundary layer to just above the base of the mixed layer to be consistent with the definition of the bulk Richardson number. Reversing the order of integration and differentiation on the lefthand side of Eq. (22) gives

$$
\frac{\partial \Delta B}{\partial t}=-\left.\frac{\partial \overline{w^{\prime} b^{\prime}}}{\partial z}\right|_{h_{m^{+}}}+\Gamma_{\mathrm{ext}} \frac{\partial h_{b}}{\partial t},
$$

where it has been assumed that the buoyancy gradient just above the base of the mixed layer is zero. In the mixed layer, the flux divergence is $\partial \overline{w^{\prime} b^{\prime}} /\left.\partial z\right|_{h_{m^{+}}} \approx-$ $\overline{w^{\prime} b_{m}^{\prime}} / h_{m}$.

In Eq. (21), the mixed layer current represents the change in current across the stratified shear layer. The equation governing the current shear can also be integrated from just below the base of the boundary layer to just above the base of the mixed layer to give

$$
\frac{\partial\left\langle U_{m}\right\rangle}{\partial t}=-\left.\frac{\partial \overline{u^{\prime} w^{\prime}}}{\partial z}\right|_{h_{m^{+}}},
$$

where the current shear just above the mixed layer base has been assumed to be zero.

The stress divergence just above the base of the mixed layer is

$$
\left.\frac{\partial \overline{u^{\prime} w^{\prime}}}{\partial z}\right|_{m+} \approx\left[-u_{*}^{2}+\beta u_{*}^{2}\left(1-h_{m} / h_{b}\right)\right] / h_{m^{+}}
$$

Substituting Eqs. (23) and (24) into Eq. (21) gives

$$
\begin{aligned}
\frac{\partial h_{m}}{\partial t}= & -\frac{\overline{w^{\prime} b^{\prime}}}{\Delta B} \frac{\left(h_{b}-h_{m}\right)}{h_{b}}+\left\{\left[1+\frac{\Gamma_{\mathrm{ext}}\left(h_{b}-h_{m}\right)}{\Delta B}\right]\right. \\
& \left.-\frac{2}{A_{b}}\left[(1-\beta) \frac{h_{b}}{h_{m}}+\beta\right] \frac{\mathrm{Ri}_{\mathrm{cr}}}{\mathrm{Ri}_{b}}\right\} \frac{\partial h_{b}}{\partial t},
\end{aligned}
$$

where Eq. (18) has been used to express the stress divergence at the base of the mixed layer in terms of $\partial h_{b} / \partial t$.

The first term on the right-hand side of Eq. (18) increases the mixed layer depth and is analogous to the usual entrainment velocity in normal mixed layer models. However, it is modified by the ratio of the depth of the stratified shear layer to the depth of the boundary layer, which increases with time. The second term is proportional to the rate of deepening of the boundary layer. The expression in the square brackets can be positive or negative, so the second term in Eq. (26) can lead to either shoaling or deepening of the mixed layer.

Figures $17 \mathrm{a}-\mathrm{c}$ show the mixed layer depths calculated from Eq. (26) compared to the mixed layer depths diagnosed from the LES. For all of the simulations, the calculated variation in the mixed layer depth is much less than the variation in the boundary layer depth, and overall the agreement between $\partial h_{m} / \partial t$ calculated from Eq. (26) and the results from the LES are reasonable.

The small variation in the mixed layer depth with time is not a trivial result, because it requires that the two terms in Eq. (26) are either both small or tend to cancel. For these simulations, the variation in $h_{m}$ is small because both terms on the right-hand side of Eq. (26) are small. However, although the second term is small, the individual terms within the parenthesis are $\approx 2$ and therefore cancel.

The second term, in the brackets, in Eq. (26) acts to maintain the bulk Richardson number at the critical value. For example, if $\mathrm{Ri}_{b}>\mathrm{Ri}_{\text {cr }}$, this term is reduced so that the whole term leads to an increase in $\partial h_{m} / \partial t$ relative to $\partial h_{b} / \partial t$. The decrease in the thickness of the shear layer reduces the Richardson number.

Equation (26) indicates that the mixed layer depth depends on the external stratification. However, as discussed previously for the present simulations, $\Gamma_{\text {ext }}\left(h_{b}-\right.$ $\left.h_{m}\right) / \Delta B \approx 1$, so this sensitivity is hidden. The change in mixed layer buoyancy is relatively small in these simulations; however, this change would be larger if the mixed layer was also cooled through surface fluxes. This additional cooling would lead to $\Gamma_{\text {ext }}\left(h_{b}-h_{m}\right) / \Delta B>1$, which would tend to increase $h_{m}$. The first term in Eq. (26) would also increase $h_{m}$. This suggests that a constant mixed layer depth is only expected to occur if surface buoyancy forcing is sufficiently small.

\section{Discussion}

Figure 19 summarizes the main results of this study by showing the pathways by which energy input from the wind produces turbulence. The energy input at the surface is partitioned into two components, one associated with the mean mixed layer velocity and the other with the additional surface current that arises from the presence of mean velocity gradients in the mixed layer. This partitioning of the surface kinetic energy flux is useful because it allows the depths over which the components of the surface kinetic energy flux vary to be different, reflecting the different length scales for the turbulent processes. 


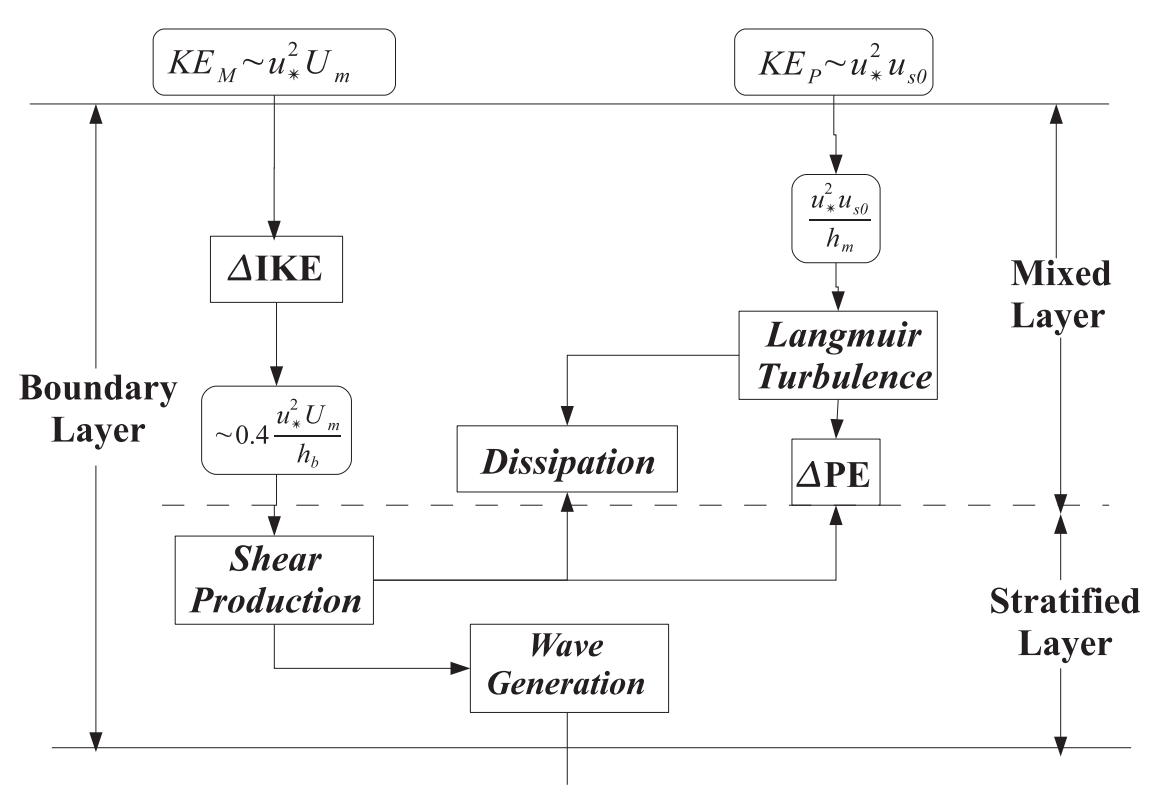

FIG. 19. A schematic showing the energy pathways in the wind-driven boundary layer.

Mixed layer turbulence results from the kinetic energy flux associated with presence of current shear in the mixed layer. This component of the surface kinetic energy flux goes to zero over the depth of the mixed layer, and in these simulations this drives the Langmuir turbulence. The shear turbulence is attributed to the component of the surface energy flux associated with the mean mixed layer velocity, which decreases to zero over the depth of the boundary layer. As shown, this component of the surface energy flux generates turbulence within both the mixed layer and the stratified shear layer.

The results from this study have significant implications for parameterization of the upper ocean boundary layer. First, there are two turbulent processes with different velocity and length scales that must be parameterized. Current parameterizations of the upper ocean assume that only a single velocity and length scale is needed to characterize the boundary layer turbulence (e.g., Large et al. 1994). To determine the turbulence length scales, the depth of the boundary layer and mixed layer must be determined. In a scheme such as the KPP (Large et al. 1994), the diagnosis of the boundary layer depth is done with a bulk Richardson number. However, Eq. (20) shows that the Richardson number is not constant but depends on the depth of the mixed layer and boundary layer through the nondimensional parameter $\zeta$.

An expression for the rate of change of the boundary layer depth was obtained from the bulk model [Eq. (18)]. The magnitude of $\partial h_{b} / \partial t$ given by Eq. (18) is less than $1 \%$ of the turbulent velocity scale $\nu_{*}$. This implies that it is reasonable to treat the turbulence as quasi steady, because $h_{b}$ will change by only a small amount over the eddy turnover time scale $h_{b} / \nu_{*}$. This time-scale separation justifies the use of first-order closures for parameterization schemes. However, it is not clear that Eq. (18) can be reduced to a diagnostic expression for the boundary layer depth, such as that used in the KPP scheme (Large et al. 1994), and in using the present results it may be necessary to treat the boundary layer depth as a prognostic variable. It is also not clear how the present results could be incorporated into higherorder closures, such as TKE schemes.

Figure 19 includes the generation of waves in the shear layer, which has not been discussed. Figures 20a,b show the components of the transport term in the TKE budget in the shear layer. The turbulent transport term (Fig. 20a) shows TKE being transported from the region around the base of the mixed layer to the base of the boundary layer. The pressure term (Fig. 20b) is negative throughout the shear layer, which implies a loss of energy from the boundary layer through waves. These waves transport momentum and cause the damping layer at the base of the model, where they are absorbed, to accelerate. Although only a small fraction of the total energy is converted to waves, the magnitude of the sink of TKE is comparable to that due to buoyancy. Given that the simulations used in this study were deliberately simplified, to what extent are the results obtained likely to be relevant to the real ocean?

The simulations provide an approximation to the resonant wind-driven mixing described by Large and 

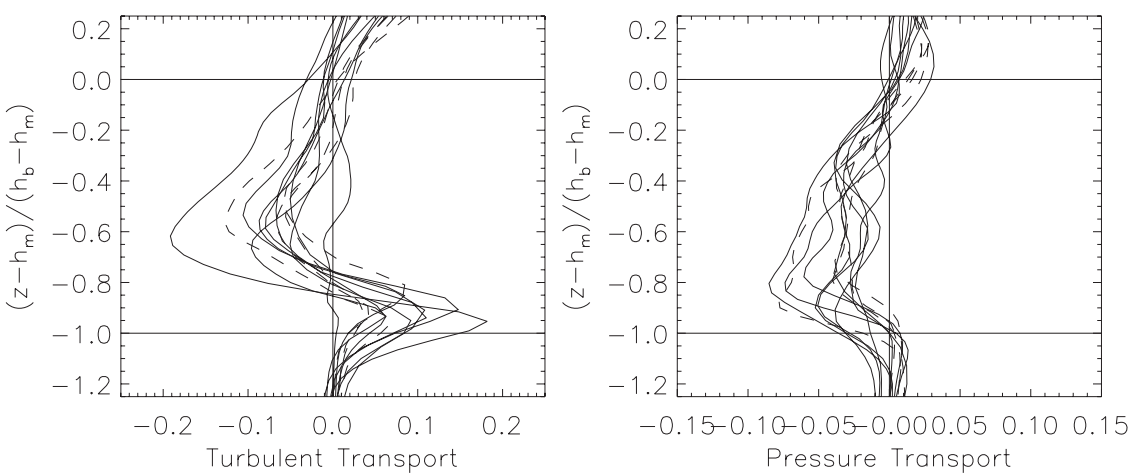

FIG. 20. Profiles of the components of the transport term in the stratified layer: (a) turbulent and (b) pressure transport. The profiles are scaled by $\nu_{*}^{3} / h_{b}$. (a) Turbulent transport. Solid curve is simulation A, dashed curve is simulation $\mathrm{B}$, and dashed-dotted-dotted-dotted curve is simulation $\mathrm{C}$.

Crawford (1995), because the surface stress and mean currents remain aligned. Qualitatively, the results presented here are consistent with those obtained by Skyllingstad et al. (2000).

Dohan and Davis (2010) contrast the response of the upper ocean boundary layer to two storms: one resonant and the other not. They found that, in the resonant storm, the mixed layer did not deepen but the boundary layer did and the gradient Richardson number in the stratified layer decreased to between 0.2 and 0.4. At the height of the resonant storm, the estimated stress profile was approximately linear as in the present simulations.

Skyllingstad et al. (2000) suggested that the closest counterpart to the present boundary layer flow was that of a weakly stratified stable boundary layer in the atmosphere. However, in the atmospheric case, the boundary layer turbulence would be characterized by a single velocity and length scale, whereas that is not the case for the boundary layer studied here. The boundary layer described here and by Skyllingstad et al. (2000) appears to be unique to the upper ocean.

The present simulations do not correspond to the situation in the eastern Pacific in that they lack the shear associated with the equatorial undercurrent. However, the results do provide a connection between the surface forcing and turbulence below the mixed layer.

Turbulence dissipation has been measured at $140^{\circ} \mathrm{W}$ during a number of observational campaigns, which are summarized in Lien et al. (1995). These observations show that the deep cycle turbulence responds to the wind stress, being weak when the surface stress is small and stronger with larger surface stresses. The scaling developed here would suggest that the generation of turbulence will also depend on the surface current; in particular, if the surface current and surface stress are opposed, then the mechanism described here will not operate. Lien et al. (1995) note that deep cycle turbulence was weak in December 1991, even though winds were strong. During the preceding November, surface currents had been westward with strong easterly winds, and during this period the deep cycle turbulence was strong. However, in December the surface current became eastward, whereas the winds remained strong easterlies. The deep cycle turbulence was much weaker but not completely absent, as the present results would suggest. This weakened deep cycle turbulence might be associated with the generation of waves through the effects of convective plumes impacting at the base of the mixed layer, which is suggested by Wijesekera and Dillon (1991) as a possible mechanism for generating deep cycle turbulence.

Wang and Müller (2002) used LES to investigate the role of shear instability on deep cycle turbulence. They found that strong turbulence and wave activity was associated with shear around the base of the mixed layer. The wavelength of these waves was dependent on the presence of the equatorial undercurrent shear. Wang and Müller (2002) concluded that shear at the base of the mixed layer and the equatorial undercurrent shear were both important in deep cycle turbulence and waves. Without the mixed layer shear, convective forcing alone was much less effective at generating waves and turbulence.

The most characteristic feature of the deep cycle turbulence is its diurnal variation. The picture developed here explains this as resulting from the stabilization of the boundary layer during the day cutting off the transport of mean kinetic energy into the deep cycle layer (Townsend 1976). Without the surface forcing, the turbulence decays.

It seems likely that the results described here are of relevance to the problem of deep cycle turbulence, even 
if they are not the complete story. It would be useful to carry out further simulations including the shear associated with the equatorial undercurrent to help clarify the relationship between the shear turbulence the observed long waves and the surface forcing.

This study has been based on large-eddy simulations of a particularly simple situation to enable a detailed analysis of the turbulent processes to be made. However, to apply this work in developing improved parameterizations, for example, the present study needs to be extended to more complex situations. The most immediate need is to consider situations with nonzero Coriolis parameter to understand how the sub-mixed layer processes interact with inertial shear. Beyond this, the effects of surface buoyancy fluxes and the interaction with existing shear below the mixed layer (e.g., due to geostrophic shear) need to be investigated.

\section{Summary}

This study has investigated the structure of the upper ocean boundary layer using large-eddy simulation. The boundary layer was forced by a constant surface stress in the absence of the Coriolis force. The resulting boundary layer has a two-layer structure, a well-mixed layer with a stratified shear layer below. Because of the absence of a Coriolis force, the shear layer deepens with time, whereas the depth of the mixed layer remains approximately constant.

In the simulations, turbulence is generated through two mechanisms. In the mixed layer, turbulence is produced through the Stokes shear to give Langmuir turbulence. Production of turbulence also occurs through current shear with the production rate being a maximum at the base of the mixed layer. The shear production extends through the full depth of the boundary layer.

The key results from this study are as follows:

- The turbulence in the mixed layer scales has the characteristics of Langmuir turbulence described by Grant and Belcher (2009).

- The production of turbulence through current shear scales as $u_{*}^{2}\left\langle U_{m}\right\rangle / h_{b}$.

- The length scale for the mixed layer turbulence is the mixed layer depth, whereas for the shear turbulence the length scale is boundary layer depth $h_{b}$.

- The velocity scale for the shear turbulence is $\nu_{*} \sim\left(u_{*}^{2}\left\langle U_{m}\right\rangle\right)^{1 / 3}$.

- Exchanges of heat and momentum across the base of the mixed layer can be described in terms of an eddy diffusivity, which scales as $\nu_{*} h_{b}$.

- A simple bulk model of the boundary layer based on the LES results suggests that the deepening of the boundary layer is not dependent on stratification, whereas the mixed layer depth remains approximately constant if surface buoyancy forcing is small.

The scaling for the shear turbulence can be interpreted as the divergence of a mean kinetic energy flux. The present results should help improve parameterizations of the wind-driven upper ocean boundary layer for use in large-scale models.

Acknowledgments. This work was supported by the Natural Environment Research Council (NERC Research Grant NE/DO10810/1).

\section{REFERENCES}

Brown, A. R., S. H. Derbyshire, and P. J. Mason, 1994: Large-eddy simulation of stable atmospheric boundary layers with a revised stochastic subgrid model. Quart. J. Roy. Meteor. Soc., 120, 1485-1512.

Craik, A. D. D., and S. Leibovich, 1976: A rational model for Langmuir circulations. J. Fluid Mech., 73, 401-426.

Dohan, K., and R. E. Davis, 2010: Mixing in the transition layer during two storm events. J. Phys. Oceanogr., 32, 42-66.

Garwood, R. W., 1977: An oceanic mixed layer model capable of simulating cyclic states. J. Phys. Oceanogr., 7, 455-468.

Grant, A. L. M., 1992: The structure of turbulence in the nearneutral atmospheric boundary layer. J. Atmos. Sci., 49, 226-239.

— lence in the ocean mixed layer. J. Phys. Oceanogr., 39, 18711887.

Gregg, M. C., H. Peters, J. C. Wesson, N. S. Oakey, and T. J. Shay, 1985: Intensive measurements of turbulence and shear in the equatorial undercurrent. Nature, 318, 140-144.

Hunt, J. C. R., J. C. Kaimal, and J. E. Gaynor, 1985: Some observations of turbulence structure in stable layers. Quart. J. Roy. Meteor. Soc., 111, 793-815.

Johnston, T. M. S., and D. L. Rudnick, 2009: Observations of the transition layer. J. Phys. Oceanogr., 39, 780-797.

Kantha, L. H., and C. A. Clayson, 1994: An improved mixed layer model for geophysical applications. J. Geophys. Res., 99, $25235-25266$.

Kraus, E. B., and J. S. Turner, 1967: A one-dimensional model of the seasonal thermocline, Part II: The general theory and its consequences. Tellus, 9, 98-105.

Large, W. G., and G. B. Crawford, 1995: Observations and simulations of upper-ocean response to wind events during the Ocean Storms experiment. J. Phys. Oceanogr., 25, 2831-2852.

_ equatorial ocean model using large eddy simulations and observations. J. Phys. Oceanogr., 29, 449-464.

— J. J. McWilliams, and S. C. Doney, 1994: Oceanic vertical mixing: A review and a model with a nonlocal boundary layer parameterization. Rev. Geophys., 32, 363-403.

Lentz, S. J., 1992: The surface boundary layer in coastal upwelling regions. J. Phys. Oceanogr., 22, 1517-1539.

Lien, R.-C., D. R. Caldwell, M. C. Gregg, and J. N. Moum, 1995: Turbulence variability at the equator in the central Pacific at the beginning of the 1991-1993 El Niño. J. Geophys. Res., 100 (C4), 6881-6898. 
E. A. D'Asaro, and M. J. McPhaden, 2002: Internal waves and turbulence in the upper central equatorial Pacific: Lagrangian and Eulerian observations. J. Phys. Oceanogr., 32, 2619-2639.

McWilliams, J. C., P. P. Sullivan, and C. H. Moeng, 1997: Langmuir turbulence in the ocean. J. Fluid Mech., 334, 1-30.

Mellor, G., 2001: One-dimensional, ocean surface layer modeling: A problem and a solution. J. Phys. Oceanogr., 31, 790-809.

Moum, J. N., D. Herbert, C. A. Paulson, and D. R. Caldwell, 1992 Turbulence and internal waves at the equator. Part I: Statistics from towed thermistors and a microstructure profiler. J. Phys. Oceanogr., 22, 1330-1345.

—, R. C. Lien, A. Perlin, J. D. Nash, M. C. Gregg, and P. J. Wiles, 2009: Sea surface cooling at the equator by subsurface mixing in tropical instability waves. Nat. Geosci., 2, 761-765, doi:10.1038/ngeo657.

Nieuwstadt, F. T. M., 1984: The turbulent structure of the stable, nocturnal boundary layer. J. Atmos. Sci., 41, 2202-2216.

Niller, P. P., and E. B. Kraus, 1977: One dimensional models of the upper ocean. Modeling and Prediction of the Upper Layers of the Ocean, E. B. Kraus, Ed., Pergamon Press, 143-172.

Otte, M. J., and J. C. Wyngaard, 2001: Stably stratified interfaciallayer turbulence from large-eddy simulation. J. Atmos. Sci., 58, 3424-3442.

Panofsky, H., and J. Dutton, 1984: Atmospheric Turbulence, Models and Methods for Engineering Applications. Wiley International, $429 \mathrm{pp}$.

Pearson, H. J., J. S. Puttock, and J. C. R. Hunt, 1983: A statistical model of fluid element motions and vertical diffusion in homogeneous stratified fluid. J. Fluid Mech., 129, 219-249.

Piacsek, S. A., and G. P. Williams, 1970: Conservation properties of convection difference schemes. J. Comput. Phys., 6, 392-405.

Polton, J. A., and S. E. Belcher, 2007: Langmuir turbulence and deeply penetrating jets in an unstratified mixed layer. J. Geophys. Res., 112, C09020, doi:10.1029/2007JC004205.

Price, J. F., R. A. Weller, and R. Pinkel, 1986: Diurnal cycling: Observations and models of the upper ocean response to diurnal heating, cooling and wind mixing. J. Geophys. Res., 91, $8411-8427$.
Rudnick, D. L., 2003: Observations of momentum transfer in the upper ocean. Did Ekman get it right? Near-Boundary Processes and Their Parameterization: Proc. 'Aha Huliko'a Winter Workshop, Honolulu, HI, University of Hawaii at Manoa, 163-170.

Shutts, G. J., and M. E. B. Gray, 1994: A numerical modelling study of the geostrophic adjustment process following deep convection. Quart. J. Roy. Meteor. Soc., 120, 1145-1178.

Skyllingstad, E. D., W. D. Smyth, and G. B. Crawford, 2000: Resonant wind-driven mixing in the ocean boundary layer. J. Phys. Oceanogr., 30, 1866-1890.

Sullivan, P. P., C.-H. Moeng, B. Stevens, D. H. Lenschow, and S. D. Mayor, 1998: Structure of the entrainment zone capping the convective atmospheric boundary layer. J. Atmos. Sci., 55, 3042-3064.

Sun, C., W. D. Smyth, and J. N. Moum, 1998: Dynamic instability of stratified shear flow in the upper equatorial Pacific. J. Geophys. Res., 103, 10 323-10 337.

Tennekes, H., and J. L. Lumley, 1972: A First Course in Turbulence. Cambridge University Press, $372 \mathrm{pp}$.

Thorpe, S. A., 2004: Langmuir circulation. Annu. Rev. Fluid, 36, 55-79.

Townsend, A. A., 1976: The Structure of Turbulent Shear Flow. Cambridge University Press, $429 \mathrm{pp}$.

Wang, D., and P. Müller, 2002: Effects of equatorial undercurrent shear on upper-ocean mixing and internal waves. J. Phys. Oceanogr., 32, 1041-1057.

, J. C. McWilliams, and W. G. Large, 1998: Large-eddy simulation of the diurnal cycle of deep equatorial turbulence. J. Phys. Oceanogr., 28, 129-148.

Wijesekera, H. W., and T. M. Dillon, 1991: Internal waves and mixing in the upper equatorial Pacific Ocean. J. Geophys. Res., 96, 7115-7125.

Zaron, E. D., and J. N. Moum, 2009: A new look at Richardson number mixing schemes for equatorial ocean modeling. J. Phys. Oceanogr., 39, 2652-2664.

Zedler, S. E., T. D. Dickey, S. C. Doney, J. F. Price, Y. You, and G. L. Mellor, 2002: Analyses and simulations of the upper ocean's response to Hurricane Felix and the Bermuda Testbed Mooring site: 13-23 August 1995. J. Geophys. Res., 107, 3232, doi:10.1029/2001JC000969. 